TRANSACTIONS OF THE

AMERICAN MATHEMATICAL SOCIETY

Volume 361, Number 2, February 2009, Pages 819-848

S 0002-9947(08)04709-0

Article electronically published on September 29, 2008

\title{
DISINTEGRATING TENSOR REPRESENTATIONS OF NILPOTENT LIE GROUPS
}

\author{
JAWHAR ABDENNADHER AND JEAN LUDWIG
}

\begin{abstract}
Let $G$ be a simply connected nilpotent Lie group and $H$ a closed connected subgroup of $G$. Given an irreducible unitary representation $\pi$ of $G$, we present an explicit disintegration of the restriction $\pi_{\mid H}$ of $\pi$ to $H$. Such a disintegration relies on the description of the double cosets space $H \backslash G / B$ for an arbitrary closed connected subgroup $B$ of $G$, and the well-known smooth disintegration of monomial representations of nilpotent Lie groups. As an application we get a concrete disintegration and a criterion of irreducibility for tensor products of a finite number of irreducible representations of $G$.
\end{abstract}

\section{INTRODUCTION}

In this paper, we present a concrete disintegration of the tensor product of two irreducible unitary representations of a simply connected, connected nilpotent Lie group $G=\exp \mathfrak{g}$ into irreducibles. "Concrete" here means that we write down an explicit integral of the spaces of isotopic components involved in the disintegration and an explicit unitary intertwining operator which realizes such a decomposition.

Towards that purpose, we use the well-known fact that a tensor product of two irreducible representations $\pi, \pi^{\prime}$ of $G$ corresponds to the restriction to the diagonal group $D$ of the irreducible representation $\pi \times \pi^{\prime}$ of the group $G \times G$. We are then led to study the general problem of describing a concrete disintegration of the restriction of an irreducible unitary representation of $G$ to a closed connected subgroup $H=\exp \mathfrak{h}$. The paper is organized as follows: we start by recording some basic results of the representation theory of Lie groups. We especially insist on the structure of nilpotent Lie groups and we introduce in section 2 some notation and conventions useful for the sequel of the paper. Using the orbit method, one realizes any $\pi \in \hat{G}$ as a monomial representation $\pi=\pi_{\ell, \mathfrak{b}}=\operatorname{ind}_{B}^{G} \chi_{\ell}$, where $\ell \in \mathfrak{g}^{*}$ and where the Lie algebra $\mathfrak{b}$ of $B$ stands for a polarizing subalgebra of $\mathfrak{g}$ at $\ell$. It comes out therefore from Mackey ([11) that the restriction of $\pi$ to $H$ is disintegrated over the set of double cosets space $H \backslash G / B$ and that any isotopic component is of the form $\operatorname{ind}_{B(x)}^{H} \chi_{\ell(x)}$, where $B(x)=H \cap \psi(x) B \psi(x)^{-1}, x \in H \backslash G / B, \ell(x)=$ $\operatorname{Ad}^{*}(\psi(x)) \ell_{\mid \mathfrak{h}}$ and $\psi: H \backslash G / B \rightarrow G$ is a section for the double cosets space. So,

Received by the editors March 16, 2007.

2000 Mathematics Subject Classification. Primary 22E27.

(C)2008 American Mathematical Society Reverts to public domain 28 years from publication 
the main issue is to provide a comprehensive description of the double cosets space and accurate information on its structural features that the disintegration of the restriction strongly relies on. This will be the aim of section $\underline{3}$, where we provide a description of an open dense subset of $H \backslash G / B$ and of such a section $\psi$ which gives an explicit decomposition of $\pi_{\mid H}$ in term of an integral over $H \backslash G / B$ of the representations $\operatorname{ind}_{B(x)}^{H} \chi_{\ell(x)}$ (see Proposition 3.27). The fourth section is devoted to recalling the results of Baklouti-Ludwig [2] concerning an explicit disintegration of monomial representations. These results will be used as a fundamental genesis to obtain our concrete disintegration of the restriction of $\pi$ to $H$. In Section 5. we apply our construction to the disintegration of the tensor product of two irreducible representations $\pi$ and $\pi^{\prime}$. The disintegration is made over a subvariety of $\mathfrak{g}^{*}$, which is contained in the sum of the Kirillov orbits of $\pi$ and $\pi^{\prime}$. We show in section 6 through an example that the formulae describing the disintegration strongly depend on the choice of the polarizations used to realize the representations $\pi$ and $\pi^{\prime}$. Finally, the last section is devoted to providing necessary and sufficient conditions relying on the representations $\pi$ and $\pi^{\prime}$ for the representation $\pi \otimes \pi^{\prime}$ to be irreducible.

It is noteworthy to point out in this context that an "abstract" disintegration into irreducibles for restrictions of unitary representations to connected closed subgroups of simply connected nilpotent Lie groups has been given in [5]. Abstract here means that the measure class in $\hat{G}$ for the disintegration of the restriction and the multiplicities of irreducibles appearing in the disintegration is given. In [7] these results are extended to encompass all exponential solvable Lie groups. In [3], another concrete disintegration of the restriction into irreducibles to closed connected subgroups is given.

\section{PREliminaries AND NOtATion}

Let $\mathfrak{g}$ be a nilpotent Lie algebra, $\mathfrak{b}$ any subalgebra and $G \supset B$ their simply connected Lie groups. Given a flag of ideals

$$
\mathfrak{g}_{n+1}:=\{0\} \varsubsetneqq \cdots \varsubsetneqq \mathfrak{g}_{i} \varsubsetneqq \cdots \varsubsetneqq \mathfrak{g}_{1}=\mathfrak{g}, \operatorname{dim}\left(\mathfrak{g}_{i} / \mathfrak{g}_{i+1}\right)=1,
$$

we let $G_{i}:=\exp \mathfrak{g}_{i}$ for every $i=1, \cdots, n$ and we choose a vector $Z_{i} \in \mathfrak{g}_{i} \backslash \mathfrak{g}_{i+1}$, so that $\mathfrak{g}_{i}=\operatorname{span}\left\langle Z_{i}, \cdots, Z_{n}\right\rangle$. We obtain in this way a Jordan-Hölder basis $\mathcal{Z}=$ $\left(Z_{1}, \cdots, Z_{n}\right)$ of $\mathfrak{g}$. Recall that the exponential mapping $\exp : \mathfrak{g} \longrightarrow G$ is a diffeomorphism, so, just for brevity, we denote by $V_{1} \cdot V_{2} \ldots V_{k}:=\exp \left(V_{1}\right) \exp \left(V_{2}\right) \ldots \exp \left(V_{k}\right) \in$ $G$, for any $k$ vectors $V_{1}, \cdots, V_{k} \in \mathfrak{g}$.

Let $d g$ denote the Haar mesure on $G$, that is, for any $f \in L^{1}(G)$, one has

$$
\int_{G} f(g) d g=\int_{\mathbb{R}^{n}} f\left(z_{1} Z_{1} \ldots z_{n} Z_{n}\right) d z .
$$

Since $G$ is nilpotent, the quotient space $G / B$ has a $G$-invariant measure which is unique up to a positive scalar multiple. To describe such a measure, which we shall denote by $d \dot{g}$, we proceed as follows. Let

$$
\mathcal{I}^{\mathfrak{g} / \mathfrak{b}}=\left\{k \in\{1, \cdots, n\}, Z_{k} \notin \mathfrak{b}+\mathfrak{g}_{k+1}\right\}=:\left\{k_{1}<\cdots<k_{p}\right\} .
$$

We obtain the sequence of subalgebras

$$
\mathfrak{b}^{p+1}:=\mathfrak{b} \varsubsetneqq \cdots \varsubsetneqq \mathfrak{b}^{j}:=\mathbb{R} Z_{k_{j}} \oplus \mathfrak{b}^{j+1} \varsubsetneqq \cdots \varsubsetneqq \mathfrak{b}^{1}=\mathfrak{g}
$$


and the Malcev basis $\mathcal{M}:=\left(Z_{k_{1}}, \cdots, Z_{k_{p}}\right)$ of $\mathfrak{g}$ relative to $\mathfrak{b}$. The invariant measure $d \dot{g}$ is then given by

$\mu_{\mathcal{M}}(\varphi)=\mu_{\mathfrak{g} / \mathfrak{b}}(\varphi)=\int_{G / B} \varphi(\dot{g}) d \dot{g}:=\int_{\mathbb{R}^{p}} \varphi\left(\zeta_{1} Z_{k_{1}} \ldots \zeta_{p} Z_{k_{p}} \cdot B\right) d \zeta, \varphi \in \mathcal{C}_{c}(G / B)$,

where $\mathcal{C}_{c}(G / B)$ denotes the space of complex-valued continuous functions with compact support on $G / B$. This is a consequence of the fact that the mapping

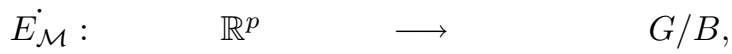

$$
\begin{aligned}
& \zeta=\left(\zeta_{1}, \cdots, \zeta_{p}\right) \longmapsto \zeta_{1} Z_{k_{1}} \ldots \zeta_{p} Z_{k_{p}} \cdot B=: \dot{E_{\mathcal{M}}}(\zeta)
\end{aligned}
$$

is a diffeomorphism (for detailed explanations see [6]).

If $\mathfrak{h}$ is another subalgebra of $\mathfrak{g}$, then we denote by $\mathcal{I}^{\mathfrak{h}} \subset\{1, \cdots, n\}$ the index set

$$
\mathcal{I}^{\mathfrak{h}}:=\left\{i \in\{1, \cdots, n\} ; \mathfrak{h}+\mathfrak{g}_{i+1}=\mathfrak{h}+\mathfrak{g}_{i}\right\}=\{1, \cdots, n\} \backslash \mathcal{I}^{\mathfrak{g} / \mathfrak{h}} .
$$

We can then assume, without any loss of generalities, that the vectors $Z_{i}, i \in \mathcal{I}^{\mathfrak{h}}$, are contained in $\mathfrak{h}$, so that

$$
\mathfrak{h}=\operatorname{span}\left\{Z_{i}, i \in \mathcal{I}^{\mathfrak{h}}\right\} .
$$

Definition 2.1. Let $B=\exp \mathfrak{b}$ be a closed connected subgroup of $G$ and let $\left(\rho, \mathcal{H}_{\rho}\right)$ be a unitary representation of $B$. We define the induced representation $\tau_{\rho}:=\operatorname{ind}_{B}^{G} \rho$ of $G$ as follows. Let

$$
\begin{aligned}
C_{c}(G / B, \rho)= & \left\{\xi: G \rightarrow \mathcal{H}_{\rho}, \xi \text { continuous with compact support modulo } B,\right. \\
& \left.\xi(g b)=\rho(b)^{-1}(\xi(g)) \text { for all } g \in G, b \in B\right\} .
\end{aligned}
$$

We define a scalar product on $C_{c}(G / B, \rho)$ by

$$
\langle\xi, \eta\rangle_{\mathcal{H}}:=\int_{G / B}\langle\xi(x), \eta(x)\rangle_{\mathcal{H}_{\rho}} d x, \xi, \eta \in C_{c}(G / B, \rho)
$$

(where $\langle,\rangle_{\mathcal{H}_{\rho}}$ stands for the scalar product on $\mathcal{H}_{\rho}$ ) and therefore a Hilbert norm

$$
\|\xi\|_{2}^{2}=\langle\xi, \xi\rangle_{\mathcal{H}}=\int_{G / B}\langle\xi(x), \xi(x)\rangle_{\mathcal{H}_{\rho}} d \dot{x} .
$$

Let us define the left translation $\lambda(g)$ on $C_{c}(G / B, \rho)$ by

$$
\lambda(g) \xi\left(g^{\prime}\right)=\xi\left(g^{-1} g^{\prime}\right), \xi \in C_{c}(G / B, \rho), g, g^{\prime} \in G .
$$

It is easy to see that $\|\lambda(g) \xi\|_{2}=\|\xi\|_{2}$ for every $g \in G$ and $\xi \in C_{c}(G / B, \rho)$ and so $\lambda$ defines on the completion $L^{2}(G / B, \rho)$ of $C_{c}(G / B, \rho)$ a unitary representation of $G$, which will be denoted by $\tau_{\rho, B}=\operatorname{ind}_{B}^{G} \rho$ or simply by $\tau_{\rho}$. Letting $\ell \in \mathfrak{g}^{*}$ such that $\langle\ell,[\mathfrak{b}, \mathfrak{b}]\rangle=\{0\}$, we can then define the unitary character $\chi_{\ell}(\exp X):=$ $e^{-i\langle\ell, X\rangle}, X \in \mathfrak{b}$, of $B$ and form the representation $\tau_{\ell, \mathfrak{b}}:=\tau_{\chi \ell}, B$ of $G$.

\section{Remarks 2.2.}

(1) We have that the equivalence between $\tau_{\ell, \mathfrak{b}}$ is irreducible and $\mathfrak{b}$ is a polarization of $\mathfrak{g}$ in $\ell \in \mathfrak{g}^{*}$, i.e. an isotropic subalgebra of $\mathfrak{g}$ with respect to the skew-symmetric bilinear form $B_{\ell}:(X, Y) \mapsto\langle\ell,[X, Y]\rangle$ on $\mathfrak{g}$, with maximal dimension. 
(2) For a given Jordan-Hölder basis $\mathcal{Z}=\left(Z_{1}, \cdots, Z_{n}\right)$ of $\mathfrak{g}$, define the Vergne polarization of $\ell$ in $\mathfrak{g}$ as the sum

$$
\mathfrak{b}(\ell)=\sum_{i=1}^{n} \mathfrak{g}_{i}\left(\ell_{i}\right)
$$

where $\mathfrak{g}_{i}\left(\ell_{i}\right)=\left\{X \in \mathfrak{g}_{i},\left\langle\ell,\left[X, \mathfrak{g}_{i}\right]\right\rangle=0\right\}$ is the stabilizer of $\ell_{i}:=\ell_{\mid \mathfrak{g}_{i}}$ in $\mathfrak{g}_{i}=\operatorname{span}\left\langle Z_{i}, \cdots, Z_{n}\right\rangle$ for $i=1, \cdots, n$.

\section{The Set of Double cosets}

Let $G=\exp \mathfrak{g}$ be a connected, simply connected, nilpotent Lie group, $\mathfrak{b}$ and $\mathfrak{h}$ two subalgebras of $\mathfrak{g}, B=\exp \mathfrak{b}$ and $H=\exp \mathfrak{h}$ their Lie subgroups. We give a concrete description for $H \backslash G / B$, which will be used to describe later on, in subsection 3.2 an appropriate measure $d \gamma$ which appears in the disintegration of $\pi_{\mid H}$ of $\pi$ to $H$, where $\pi$ is an irreducible unitary representation of $G$ which is induced from a character $\chi_{\ell}$ of $B$.

3.1. Description of $H \backslash G / B$. For $g \in G$, we denote by $\tilde{g}$ its double coset which is the set $H \cdot g \cdot B=\{h g b,(h, b) \in H \times B\}$. Our purpose is to find an open dense subset of $H \backslash G / B$ which will support the measure $d \gamma(\tilde{g})$ and which is diffeomorphic to a Zariski open subset of $\mathbb{R}^{d}$ for some $d \in \mathbb{N}^{*}$. To illustrate this fact, we introduce the following example.

Example 3.1. Let $\mathfrak{g}$ be the 7-dimensional Lie algebra spanned by its Jordan-Hölder basis $\mathcal{Z}=\left(Z_{1}, \cdots, Z_{7}\right)$ and equipped with the nonzero brackets

$$
\left[Z_{1}, Z_{4}\right]=Z_{6}, \quad\left[Z_{1}, Z_{5}\right]=Z_{7}, \quad\left[Z_{2}, Z_{3}\right]=Z_{7} .
$$

We consider its abelian subalgebras $\mathfrak{h}=\operatorname{span}\left\langle Z_{4}, Z_{5}, Z_{7}\right\rangle$ and $\mathfrak{b}=\operatorname{span}\left\langle Z_{3}, Z_{4}, Z_{7}\right\rangle$. A routine computation shows that any element $g=: z_{1} Z_{1} \ldots z_{7} Z_{7} \in G,\left(z_{1}, \cdots, z_{7}\right) \in$ $\mathbb{R}^{7}$, can be rewritten as follows:

$$
\begin{aligned}
g & =\left(z_{1} Z_{1} \cdot z_{5} Z_{5}\right) \cdot z_{2} Z_{2} \cdot z_{6} Z_{6} \cdot\left(z_{3} Z_{3} \cdot z_{4} Z_{4} \cdot z_{7} Z_{7}\right) \\
& =\left(z_{1} z_{5} Z_{7} \cdot z_{5} Z_{5} \cdot z_{1} Z_{1}\right) \cdot z_{2} Z_{2} \cdot z_{6} Z_{6} \cdot\left(z_{3} Z_{3} \cdot z_{4} Z_{4} \cdot z_{7} Z_{7}\right) \\
& \in H \cdot z_{1} Z_{1} \cdot z_{2} Z_{2} \cdot z_{6} Z_{6} \cdot B .
\end{aligned}
$$

This implies that $\tilde{g}=H \cdot g \cdot B=H \cdot z_{1} Z_{1} \cdot z_{2} Z_{2} \cdot z_{6} Z_{6} \cdot B$.

But if $z_{1} \neq 0$, the element $z_{1} Z_{1} \cdot z_{2} Z_{2} . z_{6} Z_{6}$ can also be written as $z_{1} Z_{1} \cdot z_{2} Z_{2}$ conjugated by $\exp \left(-\frac{z_{6}}{z_{1}}\right) Z_{4}$, which is contained in $H \cap B$. So we deduce that if $z_{1} \neq 0$, then $\tilde{g}=H \cdot z_{1} Z_{1} \cdot z_{2} Z_{2} \cdot B$. As a conclusion,

$$
\begin{aligned}
H \backslash G / B & =\left\{H \cdot z_{1} Z_{1} \cdot z_{2} Z_{2} \cdot B,\left(z_{1}, z_{2}\right) \in \mathcal{V}\right\} \dot{\cup}\left\{H \cdot z_{2} Z_{2} \cdot z_{6} Z_{6} \cdot B,\left(z_{2}, z_{6}\right) \in \mathbb{R}^{2}\right\} \\
& =\mathbf{p}\left(G \backslash G_{2}\right) \cup \mathbf{p}\left(G_{2}\right),
\end{aligned}
$$

where $\mathbf{p}: g \longmapsto \tilde{g}$ is the canonical projection of $G$ on $H \backslash G / B, \mathcal{V}=\mathbb{R}^{\star} \times \mathbb{R}$ is a Zariski open subset of $\mathbb{R}^{2}$ and $G_{2}$ is the closed subgroup of $G$ with Lie algebra $\mathfrak{g}_{2}=\operatorname{span}\left\langle Z_{2}, \cdots, Z_{7}\right\rangle$.

Hence the space $H \backslash G / B$ is the disjoint union of two subsets: the first is the projection of a Zariski open subset of $G$ and the second of a Zariski closed subset. We shall see later that the measure $d \gamma(\tilde{g})$ will be supported on the first set.

We shall prove next that there exists a nonempty Zariski open subset $\mathcal{V}$ of $\mathbb{R}^{d}$ which is homeomorphic to a subset $\tilde{\mathcal{U}}$ of $H \backslash G / B$ containing the double cosets of almost all (with respect to the Haar measure) elements of $G$. 
Definitions 3.2. (1) We say that a subset $\mathcal{O}$ of $G$

- is symmetric if $\mathcal{O}=\mathcal{O}^{-1}:=\left\{g^{-1}, g \in \mathcal{O}\right\}$,

- has the property $(\mathcal{P})$ (with respect to $H$ and $B$ ) if $H \mathcal{O} B \subseteq \mathcal{O}$.

(2) It is easy to see that for any $i \in\{1, \cdots, n\}$, the subset

$$
\left\{g \in G, Z_{i} \notin \mathfrak{h}+\operatorname{Ad}(g) \mathfrak{b}+\mathfrak{g}_{i+1}\right\}
$$

either contains a Zariski open subset or its complement in $G$ does. We characterize the indices subset $\mathcal{I}(\mathfrak{g} / \mathfrak{h}, \mathfrak{g} / \mathfrak{b})$ of $\{1, \cdots, n\}$ as:

$$
i \in \mathcal{I}(\mathfrak{g} / \mathfrak{h}, \mathfrak{g} / \mathfrak{b}) \quad \Longleftrightarrow \quad\left\{\begin{array}{c}
\text { the subset } \mathcal{O}_{i}:=\left\{g \in G, Z_{i} \notin \mathfrak{h}+\operatorname{Ad}(g) \mathfrak{b}+\mathfrak{g}_{i+1}\right\} \\
=\left\{g \in G, Z_{i} \notin \operatorname{Ad}\left(g^{-1}\right) \mathfrak{h}+\mathfrak{b}+\mathfrak{g}_{i+1}\right\} \\
\text { contains a nonempty Zariski open subset of } G .
\end{array}\right.
$$

When this is the case, we denote by $\mathcal{U}_{i}$ the maximal symmetric Zariski open subset contained in $\mathcal{O}_{i}$.

(3) Also let for $i \in\{1, \cdots, n\} \backslash \mathcal{I}(\mathfrak{g} / \mathfrak{h}, \mathfrak{g} / \mathfrak{b})=: \mathcal{I}(\mathfrak{h}, \mathfrak{b})$,

$$
\mathcal{O}_{i}:=\left\{g \in G, Z_{i} \in \mathfrak{h}+\operatorname{Ad}(g) \mathfrak{b}+\mathfrak{g}_{i+1}\right\} .
$$

For $i \in \mathcal{I}(\mathfrak{h}, \mathfrak{b})$, we consider the maximal symmetric Zariski open subset $\mathcal{U}_{i}$ of $\mathcal{O}_{i}$, with the property that for every $g \in \mathcal{U}_{i}$, there exist rational mappings $G \ni g^{\prime} \mapsto h\left(g^{\prime}\right) \in \mathfrak{h}, g^{\prime} \mapsto b\left(g^{\prime}\right) \in \mathfrak{b}, g^{\prime} \mapsto b_{i+1}\left(g^{\prime}\right) \in \mathfrak{g}_{i+1}$, with no singularities in a Zariski neighbourhood $\mathcal{U}_{i}(g)$ of $g$ such that $Z_{i}=$ $h\left(g^{\prime}\right)+\operatorname{Ad}\left(g^{\prime}\right) b(g)+b_{i+1}\left(g^{\prime}\right)$ for all $g^{\prime} \in \mathcal{U}_{i}(g)$.

It is easy to see that

\section{Proposition 3.3.}

(1) For $i \in\{1, \cdots, n\}$, the following two conditions are equivalent:

- $\mathcal{U}_{i} \subset\left\{g \in G, Z_{i} \in \mathfrak{h}+\operatorname{Ad}(g) \mathfrak{b}+\mathfrak{g}_{i+1}\right\}$,

- $\mathcal{U}_{i} \subset\left\{g \in G, Z_{i} \in \mathfrak{b}+\operatorname{Ad}(g) \mathfrak{h}+\mathfrak{g}_{i+1}\right\}$.

(2) The index subset $\mathcal{I}(\mathfrak{g} / \mathfrak{h}, \mathfrak{g} / \mathfrak{b})$ is contained in $\mathcal{I}^{\mathfrak{g} / \mathfrak{h}} \cap \mathcal{I}^{\mathfrak{g} / \mathfrak{b}}=: \mathcal{I}^{\mathfrak{h}, \mathfrak{b}}$.

(3) Every $\mathcal{O}_{i}, i \in\{1, \cdots, n\}$, has the property $(\mathcal{P})$. Furthermore, the maximal Zariski open subsets $\mathcal{U}_{i}, i \in \mathcal{I}(\mathfrak{g} / \mathfrak{h}, \mathfrak{g} / \mathfrak{b})$ of $G$ do.

Proof.

(1) To find a nonempty Zariski open subset $\mathcal{U}_{i}$ of $G$ satisfying one of these inclusions, $i$ must be in $\mathcal{I}(\mathfrak{h}, \mathfrak{b})$. When this is the case, suppose that

$$
\mathcal{U}_{i} \subset\left\{g \in G, Z_{i} \in \mathfrak{b}+\operatorname{Ad}(g) \mathfrak{h}+\mathfrak{g}_{i+1}\right\} .
$$

Since $\mathcal{U}_{i}$ is symmetric, for $g \in \mathcal{U}_{i}$, we have that $Z_{i}=X+\operatorname{Ad}\left(g^{-1}\right) Y+S_{i+1}$ for some $X \in \mathfrak{b}, Y \in \mathfrak{h}$ and $S_{i+1} \in \mathfrak{g}_{i+1}$. We obtain that

$$
\operatorname{Ad}(g) Z_{i}=\operatorname{Ad}(g) X+Y+\operatorname{Ad}(g) S_{i+1}
$$

and

$$
\operatorname{Ad}(g) Z_{i}=Z_{i} \quad \bmod \mathfrak{g}_{i+1} .
$$

By putting $T_{i+1}:=\left(Z_{i}-\operatorname{Ad}(g) Z_{i}\right)+\operatorname{Ad}(g) S_{i+1} \in \mathfrak{g}_{i+1}$, we get $Z_{i}=$ $\operatorname{Ad}(g) X+Y+T_{i+1}$. Hence $Z_{i} \in \mathfrak{h}+\operatorname{Ad}(g) \mathfrak{b}+\mathfrak{g}_{i+1}$, which means that $\mathcal{U}_{i} \subset\left\{g \in G, Z_{i} \in \mathfrak{h}+\operatorname{Ad}(g) \mathfrak{b}+\mathfrak{g}_{i+1}\right\}$. Finally, as $\mathfrak{h}$ and $\mathfrak{b}$ play a symmetric role, we are done. 
(2) If $i \in\{1, \cdots, n\} \backslash \mathcal{I}^{\mathfrak{g} / \mathfrak{h}} \cap \mathcal{I}^{\mathfrak{g} / \mathfrak{b}}=\mathcal{I}^{\mathfrak{b}} \cup \mathcal{I}^{\mathfrak{h}}$, then $Z_{i} \in \mathfrak{b}+\mathfrak{g}_{i+1}$ or $Z_{i} \in \mathfrak{h}+\mathfrak{g}_{i+1}$. Therefore $\operatorname{Ad}(g)\left(Z_{i}\right) \in \mathfrak{b}+\mathfrak{g}_{i+1}$ for every $g \in G$ or $\operatorname{Ad}(g)\left(Z_{i}\right) \in \mathfrak{h}+\mathfrak{g}_{i+1}$ for every $g \in G$ and so $Z_{i} \in \operatorname{Ad}\left(g^{-1}\right) \mathfrak{b}+\mathfrak{g}_{i+1} \subset \operatorname{Ad}\left(g^{-1}\right) \mathfrak{b}+\mathfrak{h}+\mathfrak{g}_{i+1}$ for every $g \in G$ or $Z_{i} \in \operatorname{Ad}\left(g^{-1}\right) \mathfrak{h}+\mathfrak{g}_{i+1} \subset \operatorname{Ad}\left(g^{-1}\right) \mathfrak{h}+\mathfrak{b}+\mathfrak{g}_{i+1}$ for every $g \in G$. Hence $i \in \mathcal{I}(\mathfrak{h}, \mathfrak{b})$.

(3) If $Z_{i}=\operatorname{Ad}(g) X+Y+T_{i+1}=X+\operatorname{Ad}\left(g^{-1}\right) Y+S_{i+1}$ for some $X \in \mathfrak{b}, Y \in \mathfrak{h}$ and $T_{i+1}, S_{i+1} \in \mathfrak{g}_{i+1}$, then for every $(h, b) \in H \times B$, we have that

$$
Z_{i}=\operatorname{Ad}(g b)\left(\operatorname{Ad}\left(b^{-1}\right) X\right)+Y+T_{i+1}
$$

and

$$
Z_{i}=X+\operatorname{Ad}\left((h g)^{-1}\right)(\operatorname{Ad}(h) Y)+S_{i+1} .
$$

This shows that the subset of $G$ consisting of all elements $g$, such that $Z_{i} \in \operatorname{Ad}(g) \mathfrak{b}+\mathfrak{h}+\mathfrak{g}_{i+1}$ is $H$-invariant on the right and $B$-invariant on the left and so is its complement.

Remark 3.4. Putting $\mathcal{U}:=\bigcap_{i \in \mathcal{I}_{\mathfrak{h}, \mathfrak{b}}} \mathcal{U}_{i}$, we obtain a "canonical" nonempty Zariski open subset of $G$ having the property $(\mathcal{P})$, which allows us to say that

$$
\mathcal{I}(\mathfrak{g} / \mathfrak{h}, \mathfrak{g} / \mathfrak{b})=\left\{1 \leq i \leq n, \forall g \in \mathcal{U}, Z_{i} \notin \mathfrak{h}+\operatorname{Ad}(g) \mathfrak{b}+\mathfrak{g}_{i+1}\right\} .
$$

Notation 3.5. We order the set of indices $\mathcal{I}(\mathfrak{g} / \mathfrak{h}, \mathfrak{g} / \mathfrak{b})=\left\{i_{1}<\cdots<i_{d}\right\}$ and we put

$$
\mathcal{V}=\phi^{-1}(\mathcal{U}):=\left\{t \in \mathbb{R}^{d}, \phi(t) \in \mathcal{U}\right\} \quad, \quad \tilde{\mathcal{U}}=\mathbf{p}(\mathcal{U}):=\{\tilde{g}, g \in \mathcal{U}\}
$$

$$
\begin{array}{cccc}
\tilde{\phi}:=\mathbf{p} \circ \phi_{\left.\right|_{\mathcal{V}}}: & \mathcal{V} & \longrightarrow & \tilde{\mathcal{U}}, \\
& t=\left(t_{1}, \cdots, t_{d}\right) & \longmapsto & H \cdot \phi(t) \cdot B .
\end{array}
$$

(5) Finally, we provide the space of the double cosets $H \backslash G / B$ with the quotient topology; i.e. a subset $\tilde{U}$ in $H \backslash G / B$ is open if and only if the subset $U:=\mathbf{p}^{-1}(\tilde{U})$ is open in $G$. The mapping $\mathbf{p}$ is then open, since for every open subset $U$ of $G$, we have that $\mathbf{p}^{-1}(\mathbf{p}(U))=H U B=\bigcup_{h \in H, b \in B} h U b$ is open in $G$.

Lemma 3.6. In the particular case where $\mathfrak{b}$ is an ideal, we have that $\tilde{\phi}$ is a diffeomorphism between $\mathbb{R}^{d}$ and $H \backslash G / B$.

Proof. The closed subgroup $H B=B H$ has $\mathfrak{h}+\mathfrak{b}$ as Lie algebra, and it satisfies $H B \backslash G=H \backslash G / B$. Indeed for every $g \in G$,

$$
H \cdot g \cdot B=\{h g b, h \in H, b \in B\}=\left\{h b^{\prime} g, h \in H, g b g^{-1}=: b^{\prime} \in B\right\}=H B \cdot g .
$$

We also notice that $\mathcal{I}(\mathfrak{g} / \mathfrak{h}, \mathfrak{g} / \mathfrak{b})=\mathcal{I}^{\mathfrak{g} / \mathfrak{h}+\mathfrak{b}}=:\left\{Z_{i_{1}}, \cdots, Z_{i_{d}}\right\}$, since the condition $Z_{i} \notin \mathfrak{h}+\operatorname{Ad} g \mathfrak{b}+\mathfrak{g}_{i+1}$ is now equivalent to the condition $Z_{i} \notin \mathfrak{h}+\mathfrak{b}+\mathfrak{g}_{i+1}$. This tells us that $\mathcal{U}$ is all of $G$. Hence $\left(Z_{i_{1}}, \cdots, Z_{i_{d}}\right)$ is a Malcev basis for $\mathfrak{g}$ relative to $\mathfrak{h}+\mathfrak{b}$. Finally, we have the diffeomorphism: $t=\left(t_{1}, \cdots, t_{d}\right) \longmapsto H B \cdot\left(-t_{d} Z_{i_{d}}\right) \ldots\left(-t_{1} Z_{i_{1}}\right)$ between $\mathbb{R}^{d}$ and the set $H B \backslash G$ of the right classes modulo $H B$. Composed with the diffeomorphism $H B \backslash G \ni H B \cdot g^{-1} \longmapsto g \cdot H B \in G / H B$, this mapping 
becomes $\mathbb{R}^{d} \ni t=\left(t_{1}, \cdots, t_{d}\right) \longmapsto t_{1} Z_{i_{1}} \ldots t_{d} Z_{i_{d}} \cdot H B \in G / H B$, which is nothing but $\tilde{\phi}$ according to Notation $3.5(4)$. We deduce then that it is a diffeomorphic mapping.

Remark 3.7. In Theorem 3.10 we show that the canonical mapping $\tilde{\phi}: \mathcal{V} \longrightarrow \tilde{\mathcal{U}}$ is a homeomorphism. We proceed by induction on the dimension of $\mathfrak{g} / \mathfrak{b}$. We consider then the index

$$
l:=\max \left\{i \in \mathcal{I}^{\mathfrak{g} / \mathfrak{b}}\right\}
$$

and we set

$$
\mathfrak{a}:=\mathfrak{g}_{l+1}, \quad \mathfrak{c}:=\mathfrak{b}+\mathfrak{g}_{l}=\mathfrak{b} \oplus \mathbb{R} Z_{l} .
$$

Since $Z_{j} \in \mathfrak{b}$ for every $j>l$, it follows that $\mathfrak{a}$ is an ideal of $\mathfrak{g}$ contained in $\mathfrak{b}$. Furthermore

$$
\mathcal{I}^{\mathfrak{g} / \mathfrak{c}}=\mathcal{I}^{\mathfrak{g} / \mathfrak{b}} \backslash\{l\}=\left\{k_{1}<\cdots<k_{p-1}\right\},
$$

whence, by intersecting with $\mathcal{I}^{\mathfrak{g} / \mathfrak{h}}$, we obtain that $\mathcal{I}^{\mathfrak{h}, \mathfrak{c}}=\mathcal{I}^{\mathfrak{h}, \mathfrak{b}} \backslash\{l\}$.

We encounter the following two cases which must be discussed also in the proof of Lemma 3.9

a): $Z_{l} \notin \mathfrak{h}+\mathfrak{g}_{l+1}$, which means that $l \in \mathcal{I}^{\mathfrak{g} / \mathfrak{h}}$ and so $\mathcal{I}^{\mathfrak{h}, \mathfrak{b}}=\mathcal{I}^{\mathfrak{h}, \mathfrak{c}} \cup\{l\}$.

b): $Z_{l} \in \mathfrak{h}+\mathfrak{g}_{l+1}$, whence $Z_{l} \in \mathfrak{h}$ while we assume (2.3). In this case we have $l \notin \mathcal{I}^{\mathfrak{g} / \mathfrak{h}}$ and so $\mathcal{I}^{\mathfrak{h}, \mathfrak{b}}=\mathcal{I}^{\mathfrak{h}, \mathfrak{c}}$.

Since $\mathfrak{g}_{l} \subset \mathfrak{g}_{i+1}$ for all $i<l$, it follows that

$\mathfrak{h}+\operatorname{Ad}(g) \mathfrak{c}+\mathfrak{g}_{i+1}=\mathfrak{h}+\operatorname{Ad}(g)\left(\mathfrak{b}+\mathfrak{g}_{l}\right)+\mathfrak{g}_{i+1}=\mathfrak{h}+\operatorname{Ad}(g) \mathfrak{b}+\mathfrak{g}_{i+1}, \forall g \in G$.

This tells us that $\mathcal{I}(\mathfrak{g} / \mathfrak{h}, \mathfrak{g} / \mathfrak{c})=\mathcal{I}(\mathfrak{g} / \mathfrak{h}, \mathfrak{g} / \mathfrak{b}) \backslash\{l\}$.

We can also deduce from this equality that all the nonempty Zariski open subsets $\mathcal{U}_{i}^{o}\left(i \in \mathcal{I}^{\mathfrak{h}, \mathfrak{c}}\right)$ of $G$, defining the canonical Zariski open subset $\mathcal{U}^{o}=\bigcap_{\mathcal{I}_{\mathfrak{h}, \mathfrak{c}}} \mathcal{U}_{i}^{o}$ as in Remark 3.4 for $H \backslash G / C$, coincide with the $\mathcal{U}_{i}\left(i \in \mathcal{I}^{\mathfrak{h}, \mathfrak{b}} \backslash\{l\}\right)$ for $H \backslash G / B$. In particular, from $\mathcal{I}^{\mathfrak{h}, \mathfrak{b}} \supseteq \mathcal{I}^{\mathfrak{h}, \mathfrak{c}}$, we obtain that $\mathcal{U} \subseteq \mathcal{U}^{\circ}$.

Hence in the case $\mathbf{b}$ ), we have that

$$
\mathcal{I}(\mathfrak{g} / \mathfrak{h}, \mathfrak{g} / \mathfrak{b})=\mathcal{I}(\mathfrak{g} / \mathfrak{h}, \mathfrak{g} / \mathfrak{c}),
$$

since $l \notin \mathcal{I}^{\mathfrak{g}} / \mathfrak{h} \supset \mathcal{I}(\mathfrak{g} / \mathfrak{h}, \mathfrak{g} / \mathfrak{b})$ by Proposition $3.3(2)$.

In the first case, we consider the following two eventualities.

a-1) $l \in \mathcal{I}(\mathfrak{g} / \mathfrak{h}, \mathfrak{g} / \mathfrak{b})$ : This case is characterized by the existence of a nonempty Zariski open subset $\mathcal{U}_{l}$ of $G$, given by Definition $3.2(2)$, such that for every $g \in \mathcal{U}_{l}$, we have $Z_{l} \notin \mathfrak{h}+\operatorname{Ad}(g) \mathfrak{b} \quad$ (since $\mathfrak{g}_{l+1} \subseteq \operatorname{Ad}(g) \mathfrak{b}$ for $\left.g \in G\right)$.

a-2) $l \notin \mathcal{I}(\mathfrak{g} / \mathfrak{h}, \mathfrak{g} / \mathfrak{b})$ : Here we have $\mathcal{I}(\mathfrak{g} / \mathfrak{h}, \mathfrak{g} / \mathfrak{c})=\mathcal{I}(\mathfrak{g} / \mathfrak{h}, \mathfrak{g} / \mathfrak{b})$ as in the case $b)$. Hence for every $g$ in a nonempty Zariski open subset $\mathcal{U}_{l}$ of $G$, we have that $Z_{l} \in \mathfrak{h}+\operatorname{Ad}(g) \mathfrak{b}$.

Remark 3.8. For every $(w, \tilde{g}) \in G_{l} \times H \backslash G / B$, we have $H \cdot w g w^{-1} \cdot B=\tilde{g}$. This is an immediate consequence of the fact that $w g w^{-1} g^{-1} \in \exp \left(\left[\mathfrak{g}_{l}, \mathfrak{g}\right]\right) \subset \exp \left(\mathfrak{g}_{l+1}\right) \subset B$ $(g \in G)$.

Let us first show that

Lemma 3.9. The mapping

$$
\tilde{\phi}: \mathcal{V} \ni\left(t_{1}, \cdots, t_{d}\right) \longmapsto H \cdot t_{1} Z_{i_{1}} \ldots t_{d} Z_{i_{d}} \cdot B \in \tilde{\mathcal{U}}
$$

is bijective. 
Proof. We will proceed by induction on the codimension $p$ of $B$. There is nothing to prove if $p=0$ while $H \backslash G / B=\{\tilde{e}=G\}$ when $B=G$. If $p=1$, then the claim is trivial in view of Lemma 3.6. in fact, a subalgebra of codimension 1 in $\mathfrak{g}$ is an ideal of $\mathfrak{g}$. Let $l=\max \mathcal{I}^{\mathfrak{g} / \mathfrak{b}}=\left\{k_{1}<\cdots<k_{p}=l\right\}$ and $\mathfrak{c}:=\mathbb{R} Z_{l}+\mathfrak{b}$ as in Remark 3.7. We appply the induction hypothesis to $\mathfrak{g}, \mathfrak{h}$ and $\mathfrak{c}$, giving us the bijection $\widetilde{\phi}^{o}: \mathcal{V}^{o} \ni s \longmapsto H \cdot \phi^{o}(s) \cdot C \in \widetilde{\mathcal{U}}^{o}$. Let us introduce the projection

$$
\begin{array}{cccc}
\Lambda^{\circ}: \quad H \backslash G / B & \longrightarrow & H \backslash G / C, \\
& H \cdot g \cdot B=\tilde{g} & \longmapsto & \tilde{g}=H \cdot g \cdot C .
\end{array}
$$

We notice that the surjectivity of $\tilde{\phi}$ is equivalent to $H \phi(\mathcal{V}) B=\mathcal{U}$. Since we have already the first inclusion " $\subseteq$ " thanks to the property $(\mathcal{P})$, it's sufficient to prove that

$$
\{h \phi(t) b, h \in H, t \in \mathcal{V}, b \in B\} \supseteq \mathcal{U} .
$$

We now discuss the three cases of Remark 3.7.

Case a-1) $l \in \mathcal{I}(\mathfrak{g} / \mathfrak{h}, \mathfrak{g} / \mathfrak{b})=\left\{i_{1}, \cdots, i_{d}=l\right\}$.

Let's recall that $\mathcal{I}(\mathfrak{g} / \mathfrak{h}, \mathfrak{g} / \mathfrak{c})=\mathcal{I}(\mathfrak{g} / \mathfrak{h}, \mathfrak{g} / \mathfrak{b}) \backslash\{l\}=\left\{i_{1}, \cdots, i_{d-1}\right\}$, the map $\phi^{\circ}$ goes from $\mathbb{R}^{d-1}$ to $G$ and that we have the inclusion

$$
\bigcap_{i \in \mathcal{I}^{\mathfrak{h}, \mathfrak{c}}} \mathcal{U}_{i}=\mathcal{U}^{\circ} \supseteq \mathcal{U}=\mathcal{U}^{\circ} \cap \mathcal{U}_{l}
$$

By the induction hypothesis, we know that

$$
\widetilde{\phi}^{o}: \mathcal{V}^{o} \ni s=\left(t_{1}, \cdots, t_{d-1}\right) \longmapsto H \cdot \phi^{o}(s) \cdot C \in \widetilde{\mathcal{U}}^{o}
$$

is bijective. Finally, we get as in Notation 3.5(3), the nonempty Zariski open subset $\mathcal{V}^{o}=\phi^{o-1}\left(\mathcal{U}^{\circ}\right)$ of $\mathbb{R}^{d-1}$ and the open dense subset $\widetilde{\mathcal{U}}^{\circ}=\left\{\widetilde{g}=H \cdot g \cdot C, g \in \mathcal{U}^{\circ}\right\}$ of $H \backslash G / C$.

Surjectivity of $\tilde{\phi}$ :

Let $u \in \mathcal{U}=\mathcal{U}^{\circ} \cap \mathcal{U}_{l}$. As $\mathcal{U}^{\circ}=H \phi^{\circ}\left(\mathcal{V}^{\circ}\right) C$, we can write $u=h \phi^{\circ}(s) c$ for certain $h \in H, s=\left(s_{1}, \cdots, s_{d-1}\right) \in \mathcal{V}^{\circ}$ and $c \in C$. We have $c=\left(\exp \gamma Z_{l}\right) b$ where $b \in B$ and $\gamma \in \mathbb{R}$. Denoting by $t=\left(s_{1}, \cdots, s_{d-1}, t_{d}:=\gamma\right) \in \mathbb{R}^{d}$, we obtain that $u=h \phi(t) b$.

But $t \in \mathcal{V}$ since $\phi(t)=h^{-1} u b^{-1}$ is in $\mathcal{U}$ thanks to $(\mathcal{P})$. Finally $u \in H \phi(\mathcal{V}) B$.

Injectivity of $\tilde{\phi}$ :

At first, we remark that if $t=\left(t_{1}, \cdots, t_{d}\right) \in \mathbb{R}^{d}$ is in $\mathcal{V}$, then $s:=\left(t_{1}, \cdots, t_{d-1}\right) \in$ $\mathbb{R}^{d-1}$ must be in $\mathcal{V}^{o}$. Indeed, $\phi(t) \in \mathcal{U}=\mathcal{U}^{o} \cap \mathcal{U}_{l} \subset \mathcal{U}^{o}$ and so, thanks to the property $(\mathcal{P})$, the stability of $\mathcal{U}^{\circ}$ by right multiplication with elements of $C$ gives $\phi^{o}(s)=\phi(t) \exp \left(-t_{d} Z_{l}\right) \in \mathcal{U}^{\circ}$; hence $s \in \mathcal{V}^{\circ}$.

Now let $t=\left(t_{1}, \cdots, t_{d}\right), t^{\prime}=\left(t_{1}^{\prime}, \cdots, t_{d}^{\prime}\right) \in \mathcal{V}$ such that $H \cdot \phi(t) \cdot B=H \cdot \phi\left(t^{\prime}\right) \cdot B$, whence $H \cdot \phi(s) \cdot C=H \cdot \phi\left(s^{\prime}\right) \cdot C$, where $s=\left(t_{1}, \cdots, t_{d-1}\right)$ and $s^{\prime}=\left(t_{1}^{\prime}, \cdots, t_{d-1}^{\prime}\right)$ belong to $\mathcal{V}^{\circ}$. Then $s=s^{\prime}$ while $\widetilde{\phi}^{o}$ is injective. Finally, to show that $t_{d}=t_{d}^{\prime}$, we make the following calculation. Let $h=\exp Y \in H$ and $b=\exp X \in B$, such that $\phi(t)=h \phi\left(t^{\prime}\right) b$. As a consequence of the previous discussion, we can consider the other relation $\phi\left(t^{\prime}\right)=\phi(t) \exp \left(\left(t_{d}^{\prime}-t_{d}\right) Z_{l}\right)=: u$ between these two elements of $\mathcal{U}$, 
to obtain

$$
\begin{aligned}
\phi\left(t^{\prime}\right) \exp \left(\left(t_{d}-t_{d}^{\prime}\right) Z_{l}\right) & =\exp (Y) \phi\left(t^{\prime}\right) \exp (X) \\
& \Leftrightarrow \\
\exp (-Y)\left(u \exp \left(\left(t_{d}-t_{d}^{\prime}\right) Z_{l}\right) u^{-1}\right) & =u \exp (X) u^{-1} \\
\exp (-Y) \exp \left(\operatorname{Ad}(u)\left(t_{d}-t_{d}^{\prime}\right) Z_{l}\right) & =\exp (\operatorname{Ad}(u) X) \\
& \Rightarrow \\
-Y+\left(t_{d}-t_{d}^{\prime}\right) Z_{l} & =\operatorname{Ad}(u) X \bmod \mathfrak{g}_{l+1} .
\end{aligned}
$$

In fact,

$$
\begin{aligned}
\exp \left(\operatorname{Ad}(u)\left(t_{d}-t_{d}^{\prime}\right) Z_{l}\right) & =\exp \left(\left(t_{d}-t_{d}^{\prime}\right) Z_{l}\right) \quad \bmod G_{l+1}, \\
& \Rightarrow \\
\exp (-Y) \exp \left(\operatorname{Ad}(u)\left(t_{d}-t_{d}^{\prime}\right) Z_{l}\right) & =\exp \left(-Y+\left(t_{d}-t_{d}^{\prime}\right) Z_{l}\right) \quad \bmod G_{l+1}
\end{aligned}
$$

(by the Campbell-Baker-Hausdorff formula).

But when $\left(t_{d}-t_{d}^{\prime}\right) Z_{l}=Y+\operatorname{Ad}(u) X \bmod \mathfrak{g}_{l+1}$, and if we suppose $t_{d} \neq t_{d}^{\prime}$, then $Z_{l} \in \mathfrak{h}+\operatorname{Ad}(u) \mathfrak{b}$, which contradicts the fact that $u \in \mathcal{U}$. Hence $t_{d}=t_{d}^{\prime}$ and $\tilde{\phi}$ is injective.

Case a-2) $\quad \mathcal{I}^{\mathfrak{h}, \mathfrak{b}}=\mathcal{I}^{\mathfrak{h}, \mathfrak{c}} \cup\{l\}$ but $l \notin \mathcal{I}(\mathfrak{g} / \mathfrak{h}, \mathfrak{g} / \mathfrak{b})=\left\{i_{1}, \cdots, i_{d}\right\}=\mathcal{I}(\mathfrak{g} / \mathfrak{h}, \mathfrak{g} / \mathfrak{c})$.

Here, we have $\phi^{\circ}=\phi: \mathbb{R}^{d} \longrightarrow G$, but the nonempty Zariski subsets of $\mathbb{R}^{d}$ defined by $\mathcal{V}^{\circ}=\phi^{-1}\left(\mathcal{U}^{\circ}\right)$ and $\mathcal{V}=\phi^{-1}(\mathcal{U})$ do not coincide in general. In fact, we know that $\mathcal{U}^{o}=\bigcap_{i \in \mathcal{I}^{\mathfrak{h}, c}} \mathcal{U}_{i} \supseteq \mathcal{U}=\mathcal{U}^{o} \cap \mathcal{U}_{l}$, where the Zariski open subset $\mathcal{U}_{l}$ of $G$ satisfies $\forall g \in \mathcal{U}_{l}, Z_{l} \in \mathfrak{h}+\operatorname{Ad}(g) \mathfrak{b}$. By the induction hypothesis, $\widetilde{\mathcal{U}}^{o}=\left\{\widetilde{g}=H \cdot g \cdot C, g \in \mathcal{U}^{\circ}\right\}$ is in bijection with $\mathcal{V}^{o}$ thanks to $\widetilde{\phi}^{o}$.

Surjectivity of $\tilde{\phi}$ :

Let $u \in \mathcal{U}=\mathcal{U}^{\circ} \cap \mathcal{U}_{l} \subset \mathcal{U}^{\circ}$. Then $u$ can be written as $h \phi^{\circ}(t) c=h \phi(t) c$, where $h \in H, c=\exp \left(\gamma Z_{l}\right) b \in C$ and $t \in \mathcal{V}^{\circ}$. Then also $v:=\phi(t) \exp \left(\gamma Z_{l}\right) \in \mathcal{U}$, because of property $(\mathcal{P})$. We shall show that

$$
v=h^{\prime} \phi(t) b^{\prime}
$$

for some $h^{\prime} \in H, b^{\prime} \in B$. This then shows that $t \in \mathcal{V}=\phi^{-1}(\mathcal{U})$ and so we see that $\tilde{\phi}$ is surjective.

Now since $v \in \mathcal{U}_{l}$, we have $Z_{l} \in \operatorname{Ad}\left(v^{-1}\right) \mathfrak{h}+\mathfrak{b}$. Hence there exist $Y=: \log h^{\prime} \in \mathfrak{h}$ and $X=: \log x \in \mathfrak{b}$, such that

$$
\begin{aligned}
\gamma Z_{l} & =\operatorname{Ad}\left(v^{-1}\right) Y+X \\
& \Leftrightarrow-X+\gamma Z_{l}=\operatorname{Ad}\left(v^{-1}\right) Y \\
& \Rightarrow \exp \left(\gamma Z_{l}\right) x^{-1} q=v^{-1} h^{\prime} v\left(\text { for some } q \in G_{l+1} \subset B\right) \\
& \Rightarrow \exp \left(\gamma Z_{l}\right)=v^{-1} h^{\prime} v b^{\prime \prime}\left(\text { for some } b^{\prime \prime} \in B\right) \\
& \Rightarrow v=\phi(t) \exp \left(\gamma Z_{l}\right)=\phi(t)\left(\exp \left(-\gamma Z_{l}\right) \phi(t)^{-1} h^{\prime} \phi(t) \exp \left(\gamma Z_{l}\right) b^{\prime \prime}\right) \\
& \Rightarrow v=\phi(t)\left(\phi(t)^{-1} h^{\prime} \phi(t) b^{\prime}\right)=h^{\prime} \phi(t) b^{\prime}\left(\text { for some } b^{\prime} \in B\right)
\end{aligned}
$$


Injectivity of $\tilde{\phi}$ :

This is an immediate consequence from the injectivity of $\widetilde{\phi}^{\circ}$, since $\mathcal{V} \subset \mathcal{V}^{\circ}$ and $\tilde{\phi}=\tilde{\phi}_{\mid \mathcal{V}}^{o}$.

Case b) $l \notin \mathcal{I}^{\mathfrak{g} / \mathfrak{h}}$.

This means that $Z_{l} \in \mathfrak{h}$. Now we have $\mathcal{I}(\mathfrak{g} / \mathfrak{h}, \mathfrak{g} / \mathfrak{c})=\mathcal{I}(\mathfrak{g} / \mathfrak{h}, \mathfrak{g} / \mathfrak{b})=\left\{i_{1}, \cdots, i_{d}\right\}$, $\phi^{\circ}=\phi, \mathcal{U}^{o}=\mathcal{U}=\bigcap_{i \in \mathcal{I}^{\mathfrak{h}, \mathfrak{b}}} \mathcal{U}_{i}$ and $\mathcal{V}^{\circ}=\phi^{\circ-1}\left(\mathcal{U}^{\circ}\right)=\phi^{-1}(\mathcal{U})=\mathcal{V}$. But of course the spaces $H \backslash G / B$ and $H \backslash G / C$ are different, and $\widetilde{\phi}^{\circ}$ and $\tilde{\phi}$ are related by the formula

$$
\begin{aligned}
& \widetilde{\phi}^{\circ}=\Lambda^{\circ} \circ \tilde{\phi}: \quad \mathcal{V} \quad \longrightarrow \quad \widetilde{\mathcal{U}}^{o} \\
& t=\left(t_{1}, \cdots, t_{d}\right) \longmapsto H \cdot \phi(t) \cdot C .
\end{aligned}
$$

Surjectivity of $\tilde{\phi}$ :

As we have already $\mathcal{U}=\mathcal{U}^{\circ}=H \phi^{\circ}\left(\mathcal{V}^{\circ}\right) C=H \phi(\mathcal{V}) C$, it suffices to prove the inclusion $H \phi(\mathcal{V}) C \subseteq H \phi(\mathcal{V}) B$. Now if we take $u=h \phi(t) c$ where the elements $h, t=\left(t_{1}, \cdots, t_{d}\right), c=\exp \left(\gamma Z_{l}\right) b, \gamma$ and $b$ are respectively in $H, \mathcal{V}, C, \mathbb{R}$ and $B$, then

$$
u=h \phi(t) c=h \phi(t) \exp \left(\gamma Z_{l}\right) b=\left(h \exp \left(\gamma Z_{l}\right)\right) \exp \left(-\gamma Z_{l}\right) \phi(t) \exp \left(\gamma Z_{l}\right) b,
$$

which gives

$$
\begin{aligned}
H \cdot u \cdot B & =H \cdot h \exp \left(\gamma Z_{l}\right) \exp \left(-\gamma Z_{l}\right) \phi(t) \exp \left(\gamma Z_{l}\right) b \cdot B \\
& =H \cdot \exp \left(-\gamma Z_{l}\right) \phi(t) \exp \left(\gamma Z_{l}\right) \cdot B=H \phi(t) B,
\end{aligned}
$$

by Remark 3.8 ,

Injectivity of $\tilde{\phi}$ : $\tilde{\phi}$.

This is exactly as in the case a-2); in fact, $\widetilde{\phi}^{\circ}=\Lambda^{\circ} \circ \tilde{\phi}$ is injective, and so is

Theorem 3.10. The mapping $\tilde{\phi}: \mathcal{V} \ni t=\left(t_{1}, \cdots, t_{d}\right) \longmapsto H \cdot t_{1} Z_{i_{1}} \ldots t_{d} Z_{i_{d}} \cdot B \in \tilde{\mathcal{U}}$ is a homeomorphism.

Proof. The mapping $\tilde{\phi}=\mathbf{p} \circ \phi_{\left.\right|_{\mathcal{V}}}$ is evidently continuous since this is the case for $\phi$ and $\mathbf{p}$.

Hence it is enough to prove that the mapping

$$
H \times \mathbb{R}^{d} \times B \longrightarrow G,(h, t, b) \rightarrow h \phi(t) b
$$

is open.

We do this again by induction on the dimension of $G / B$, using the notation of the proof of Lemma 3.9.

If the dimension of $G / B=1$, then $B$ is normal and we apply Lemma 3.6.

In Case a-1), it suffices to consider subsets $W_{H} \subset H$, which are open in $H$, $\left.W=W_{0} \times\right] a, b\left[\subset \mathcal{V}\right.$ which are open in $\mathbb{R}^{d-1} \times \mathbb{R}$ and subsets $W_{B} \subset B$, which are open in $B$ and to prove that $W_{H} \phi(W) W_{B}$ is open in $G$. But by the induction hypothesis for the groups $H$ and $C$, we know that

$$
W_{H} \phi(W) W_{B}=W_{B} \phi^{o}\left(W_{0}\right) \exp (] a, b\left[Z_{l}\right) W_{B}
$$

is open in $G$, since $\exp (] a, b\left[Z_{l}\right) W_{B}$ is open in $B$.

In Case a-2) consider open subsets $W_{H}$ of $\left.H, W=W_{0} \times\right] a, b[$ open in $\mathcal{V} \subset$ $\mathbb{R}^{d-1} \times \mathbb{R}$ and $W_{B}$ open in $B$ and prove that $W_{H} \phi(W) W_{B}$ is open in $G$. Let $b \in W_{B}, h \in W_{H}, t=\left(t_{1}, \cdots, t_{d}\right) \in W$. We know that $\phi=\phi_{\mid \mathcal{V}}^{o}$ and that $W$ is open in $\mathcal{V}^{o}$. Since $l \in \mathcal{I}^{\mathfrak{g} / \mathfrak{h}} \cap I^{\mathfrak{g} / \mathfrak{b}} \backslash \mathcal{I}(\mathfrak{g} / \mathfrak{h}, \mathfrak{g} / \mathfrak{b})$, there exist rational mappings

$$
g \mapsto Y(g) \in \mathfrak{h}, \quad g \mapsto X(g) \in \mathfrak{b},
$$


defined on a Zariski open neighborhood of $\phi(t)$, which we can assume to contain $W$, such that for every $g$ in this neighborhood we have that

$$
\begin{aligned}
Z_{l} & =\operatorname{Ad}\left(g^{-1}\right) Y(g)+X(g) \\
& \Rightarrow \exp \left(t_{d} Z_{l}-t_{d} X(g)\right)=g^{-1} \exp \left(t_{d} Y(g)\right) g, t_{d} \in \mathbb{R}, \\
& \Rightarrow \exp \left(t_{d} Z_{l}\right) b\left(g, t_{d}\right)^{-1}=g^{-1} \exp (t Y(g)) g \\
& \quad\left(\text { where }\left(g, t_{d}\right) \mapsto b\left(g, t_{d}\right) \in B\right. \text { is rational), } \\
& \Rightarrow \exp \left(t_{d} Z_{l}\right)=g^{-1} h\left(g, t_{d}\right) g b\left(g, t_{d}\right) \quad\left(\text { where } h\left(g, t_{d}\right)=\exp \left(t_{d} Y(g)\right) \in H\right) .
\end{aligned}
$$

Hence, for $b^{\prime} \in B, h^{\prime} \in H, t^{\prime} \in W, t_{d} \in \mathbb{R}$, we get

$$
h^{\prime} \phi\left(t^{\prime}\right) \exp \left(t_{d} Z_{l}\right) b^{\prime}=h^{\prime} h\left(\phi\left(t^{\prime}\right), t_{d}\right) \phi\left(t^{\prime}\right) b\left(g, t_{d}\right) b^{\prime} .
$$

Since the mapping

$$
\begin{array}{ccc}
H \times B \times \mathbb{R} \times W & \longrightarrow & H \times B \times \mathbb{R} \times W \\
\left(h^{\prime}, b^{\prime}, t_{d}, t^{\prime}\right) & \longmapsto & \left(h^{\prime} h\left(\phi\left(t^{\prime}\right), t_{d}\right), b\left(g, t_{d}\right) b^{\prime}, t_{d}, t^{\prime}\right)
\end{array}
$$

is a diffeomorphism, we can find a small neighbourhood $W_{H}^{\prime} \subset W_{H}$ of $h, W_{B}^{\prime} \subset W_{B}$ of $b$ and $W^{\prime} \subset W$ of $t$, such that for every $\left.t_{d} \in\right]-\varepsilon, \varepsilon[$ for some $\varepsilon>0$, we have that

$$
\left(h^{\prime} h\left(\phi\left(t^{\prime}\right), t_{d}\right), b\left(g, t_{d}\right) b^{\prime}\right) \subset W_{H} \times W_{B} .
$$

This implies by (3.2) that

$$
h \phi(t) b \in W_{B}^{\prime} \phi\left(W^{\prime}\right) \exp (]-\varepsilon, \varepsilon\left[Z_{l}\right) W_{B}^{\prime} \subset W_{H} \phi(W) W_{B} .
$$

By the induction hypothesis, we know that $W_{B}^{\prime} \phi\left(W^{\prime}\right) \exp (]-\varepsilon, \varepsilon\left[Z_{l}\right) W_{B}^{\prime}$ is an open neighborhood of $h \phi(t) b$ and thus the set $W_{H} \phi(W) W_{H}$ is open in $G$.

In Case b), we have that $Z_{l}$ is an element of $\mathfrak{c}$. We consider again open subsets $W_{H}$ of $\left.H, W=W_{0} \times\right] a, b\left[\right.$ open in $\mathcal{V} \subset \mathbb{R}^{d}$ and $W_{B}$ open in $B$ and prove that $W_{H} \phi(W) W_{B}$ is open in $G$. Let $b \in W_{B}, h \in W_{H}, t=\left(t_{1}, \cdots, t_{d}\right) \in W$. For $h^{\prime} \in H, b^{\prime} \in B, s \in \mathbb{R}$ and $t \in W$, we have that

$$
\begin{aligned}
h^{\prime} \phi(t) \exp \left(s Z_{l}\right) b^{\prime} & =h^{\prime} \exp \left(s Z_{l}\right)\left(\exp \left(-s Z_{l}\right) \phi(t) \exp \left(s Z_{l}\right)\right) b^{\prime} \\
& =h^{\prime} \exp \left(s Z_{l}\right) \phi(t) b(s, t) b^{\prime}
\end{aligned}
$$

(where $b(s, t) \in B$ is polynomial in $(s, t)$ ).

This implies as in the case a-2) that there exist small neighbourhoods $W_{H}^{\prime} \subset W_{H}$ of $h, W_{B}^{\prime} \subset W_{B}$ of $b$ and $W^{\prime} \subset W$ of $t$, such that for every $\left.s \in\right]-\varepsilon, \varepsilon[$ (for some $\varepsilon>0), h^{\prime} \in W_{H}^{\prime}, b^{\prime} \in W_{B}^{\prime}$, we have that

$$
\left(h^{\prime} h, b(s, t) b^{\prime}\right) \subset W_{H} \times W_{B} .
$$

Therefore, by (3.3) and (3.4),

$$
h \phi(t) b \in W_{B}^{\prime} \phi\left(W^{\prime}\right) \exp (]-\varepsilon, \varepsilon\left[Z_{l}\right) W_{B}^{\prime} \subset W_{H} \phi(W) W_{B}
$$

and we conclude as in the case a-2).

Notation 3.11. We define the function $\theta: H \backslash G / B \longrightarrow \mathbb{R}^{d}$ such that for every $x \in \tilde{\mathcal{U}}$, we have

$$
\theta(x)=t=\left(t_{1}, \cdots, t_{d}\right) \in \mathcal{V} \quad \Longleftrightarrow \quad x=\tilde{\phi}(t)=H \cdot t_{1} Z_{i_{1}} \ldots t_{d} Z_{i_{d}} \cdot B
$$

and we denote by

$$
\psi: H \backslash G / B \longrightarrow G
$$

the inverse of the mapping $\phi(\mathcal{V}) \longrightarrow H \backslash G / B$. Then $\psi=\phi \circ \theta$ is a continuous section of the open dense subset $\tilde{\mathcal{U}}$ of $H \backslash G / B$ into $G$. 
Example 3.12. Returning to the previous example, we can see that $\mathcal{I}^{\mathfrak{h}, \mathfrak{b}}=$ $\mathcal{I}^{\mathfrak{g} / \mathfrak{h}} \cap \mathcal{I}^{\mathfrak{g} / \mathfrak{b}}=\{1,2,6\}$ and $\mathcal{I}(\mathfrak{g} / \mathfrak{h}, \mathfrak{g} / \mathfrak{b})=\{1,2\}$. In fact, if $g=z_{1} Z_{1} \ldots z_{7} Z_{7} \in G$ (with $\left(z_{1}, \cdots, z_{7}\right) \in \mathbb{R}^{7}$ ), then $\operatorname{Ad}(g) \mathfrak{b}=\operatorname{span}\left\langle Z_{3}, Z_{4}+z_{1} Z_{6}, Z_{7}\right\rangle$, so in the Zariski open subset $\mathcal{U}_{6}:=G \backslash G_{2}$ of $G\left(z_{1} \neq 0\right)$, we have that $\forall g \in \mathcal{U}_{6}, Z_{6} \in \mathfrak{h}+\operatorname{Ad}(g) \mathfrak{b}+$ $\mathfrak{g}_{7}=\operatorname{span}\left\langle Z_{3}, Z_{4}, Z_{5}, z_{1} Z_{6}, Z_{7}\right\rangle$, but for $i=1,2$, it's clear that $Z_{i} \notin \mathfrak{h}+\operatorname{Ad}(g) \mathfrak{b}+$ $\mathfrak{g}_{i+1}, \forall g \in G=: \mathcal{U}_{i}$. In conclusion,

$$
\begin{aligned}
\mathcal{I}(\mathfrak{g} / \mathfrak{h}, \mathfrak{g} / \mathfrak{b}) & =\left\{i \in \mathcal{I}=\{1,2,6\}, Z_{i} \notin \mathfrak{h}+\operatorname{Ad}(g) \mathfrak{b}+\mathfrak{g}_{i+1} \forall g \in \mathcal{U}=G \backslash G_{2}\right\} \\
& =\{1,2\} .
\end{aligned}
$$

Now for $H \backslash G / B$, thanks to $\mathcal{V}=\phi^{-1}(\mathcal{U})=\left\{t \in \mathbb{R}^{2}, t_{1} Z_{1} \cdot t_{2} Z_{2} \in G \backslash G_{2}\right\}=$ $\mathbb{R}^{\star} \times \mathbb{R}$ and $\tilde{\mathcal{U}}=\mathbf{p}(\mathcal{U})=\left\{H \cdot z_{1} Z_{1} \ldots z_{7} Z_{7} \cdot B, z=\left(z_{1}, \cdots, z_{7}\right) \in \mathbb{R}^{\star} \times \mathbb{R}^{6}\right\}$ where these maps are given by

$$
\left(t_{1}, t_{2}\right) \longmapsto t_{1} Z_{1} \cdot t_{2} Z_{2} \quad \text { and } \quad g \longmapsto H \cdot g \cdot B
$$

we have that

$$
\begin{aligned}
& \tilde{\phi}: \quad \mathbb{R}^{\star} \times \mathbb{R} \longrightarrow \tilde{\mathcal{U}} \\
& \left(t_{1}, t_{2}\right) \longmapsto H \cdot t_{1} Z_{1} \cdot t_{2} Z_{2} \cdot B,
\end{aligned}
$$

which is a homeomorphism thanks to the previous theorem. In particular, this confirms the relation $\mathbf{p}\left(G \backslash G_{2}\right)=\tilde{\phi}\left(\mathbb{R}^{\star} \times \mathbb{R}\right)$ of the first example's calculation.

3.2. A measure on $H \backslash G / B$. Our aim is to disintegrate the restriction to a closed subgroup $H=\exp (\mathfrak{h})$ of an irreducible representation $\pi_{\ell, \mathfrak{b}}$ of $G(\mathfrak{b}=\operatorname{Lie}(B)$ is a polarization of $\mathfrak{g}$ at $\left.\ell \in \mathfrak{g}^{\star}\right)$. The first step is to realize the Hilbert space $L^{2}\left(G / B, \chi_{\ell}\right)$ as a direct integral. Therefore we need a measure $d \gamma$ on the space $H \backslash G / B$ such that

$$
L^{2}\left(G / B, \chi_{\ell}\right) \simeq \int_{H \backslash G / B}^{\oplus} L^{2}\left(H / B(x), \chi_{\ell(x)}\right) d \gamma(x),
$$

where the linear functional

$$
\ell(x)=\operatorname{Ad}^{*}(\psi(x)) \ell \quad(x \in H \backslash G / B)
$$

defines a character of the closed subgroup

$$
B(x):=H \cap \psi(x) B \psi(x)^{-1}=\exp (\mathfrak{b}(x):=\mathfrak{h} \cap \operatorname{Ad}(\psi(x)) \mathfrak{b}) .
$$

Notation 3.13. More generally, for any $g \in G$ denote by $\mathfrak{b}(g)=\mathfrak{h} \cap \operatorname{Ad}(g) \mathfrak{b}$ the Lie algebra of $B(g)=H \cap g B g^{-1}$. We may also use the notation $B(x)$ or $B(t)$ (respectively $\mathfrak{b}(x)$ and $\mathfrak{b}(t))$ when $x=\tilde{\phi}(t) \in H \backslash G / B$, instead of $B(\phi(t))$ (respectively $\mathfrak{b}(\phi(t))$.

Let $\mathcal{Y}=\left\{Y_{1}, \cdots, Y_{m}\right\}$ be a Jordan-Hölder basis of $\mathfrak{h}$. As in section 2 , let

$$
E_{\mathcal{Y}}: \mathbb{R}^{m} \longrightarrow H, \quad\left(y_{1}, \cdots, y_{m}\right) \mapsto \exp \left(y_{1} Y_{1}\right) \cdots \exp \left(y_{m} Y_{m}\right)
$$

be the diffeomorphism used to define the Haar measure $d y$ on $H$ by

$$
\int_{H} f(h) d \mathcal{Y}(h):=\int_{\mathbb{R}^{m}} f \circ E_{\mathcal{Y}}(y) d y, \quad f \in C_{c}(H) .
$$

We know that for any subalgebra $\mathfrak{k} \subset \mathfrak{h}$, if we define the index set $\mathcal{I}^{\mathfrak{h} / \mathfrak{k}}$ by

$$
\mathcal{I}^{\mathfrak{h} / \mathfrak{k}}:=\left\{i \in\{1, \cdots, m\}, Y_{i} \notin \mathfrak{k}+\mathfrak{h}_{i+1}\right\}=\left\{j_{1}<\cdots<j_{r}\right\},
$$


then the subset

$$
\mathcal{N}:=\left\{Y_{j}, j \in \mathcal{I}^{\mathfrak{h} / \mathfrak{k}}\right\}
$$

is a Malcev basis of $\mathfrak{h}$ relative to $\mathfrak{k}$ and the mapping

$$
E_{\mathcal{N}}: \mathbb{R}^{r} \longrightarrow H, \quad\left(s_{1}, \cdots, s_{r}\right) \mapsto \exp \left(s_{1} Y_{j_{1}}\right) \cdots \exp \left(s_{r} Y_{j_{r}}\right),
$$

composed with the mapping : $H \ni h \mapsto \ddot{h}=h \cdot K \in H / K$, gives the diffeomorphism

$$
\ddot{E_{\mathcal{N}}}: \mathbb{R}^{r} \longrightarrow H / K, \quad\left(s_{1}, \cdots, s_{r}\right) \mapsto \exp \left(s_{1} Y_{j_{1}}\right) \cdots \exp \left(s_{r} Y_{j_{r}}\right) \cdot K \text {. }
$$

In particular, similarly to (2.2), we obtain an $H$-invariant measure on $H / K$ denoted $d_{\mathcal{N}}(h)$ or simply $d \ddot{h}$, by putting

$$
\int_{H / K} \varphi(\ddot{h}) d_{\mathcal{N}}(h):=\int_{\mathbb{R}^{r}} \varphi \circ \ddot{E_{\mathcal{N}}}(s) d s, \quad \varphi \in C_{c}(H / K) .
$$

Proposition 3.14. There exists a Zariski open subset $\mathcal{U}^{\prime \prime}$ of $G$ with property $(\mathcal{P})$, and an index set $\mathcal{J}^{\mathfrak{h} / \mathfrak{b}(G)} \subset \mathcal{I}^{\mathfrak{h}}$, such that

$$
\mathcal{J}^{\mathfrak{h} / \mathfrak{b}(G)}=\mathcal{I}^{\mathfrak{h} / \mathfrak{h} \cap \operatorname{Ad}(g) \mathfrak{b}} \quad \text { for all } g \in \mathcal{U}^{\prime \prime} .
$$

Proof. For every $i \in \mathcal{I}^{\mathfrak{h}}$, the set

$$
\mathcal{S}_{i}^{\prime}=\left\{g \in G, Z_{i} \notin \mathfrak{h} \cap \operatorname{Ad}(g) \mathfrak{b}+\mathfrak{h} \cap \mathfrak{g}_{i+1}\right\}
$$

has property $(\mathcal{P})$ and is either contained in a Zariski closed subset of $G$ or it contains a Zariski open subset $\mathcal{S}_{i}$ with property $(\mathcal{P})$.

The collection of all the $i$ 's verifying the second eventuality gives us the subset $\mathcal{J}^{\mathfrak{h} / \mathfrak{b}(G)}$ of $\mathcal{I}^{\mathfrak{h}}$ and $\mathcal{U}^{\prime \prime}=\bigcap_{i \in \mathcal{J}_{\mathfrak{h} / \mathfrak{b}(G)}} \mathcal{S}_{i}$.

Notation 3.15. Let $\left\{j_{1}<\cdots<j_{r}\right\}$ be the elements of $\mathcal{J}^{\mathfrak{h} / \mathfrak{b}(G)}$. Then $\mathcal{N}:=$ $\left\{Y_{j_{1}}, \cdots, Y_{j_{r}}\right\}$ is a Malcev basis of $\mathfrak{h}$ relative to $\mathfrak{b}(g)$ for all $g \in \mathcal{U}^{\prime \prime}$. Now we put

$$
\mathcal{U}^{\prime}:=\mathcal{U} \cap \mathcal{U}^{\prime \prime} \subset G, \quad \mathcal{V}^{\prime}:=\phi^{-1}\left(\mathcal{U}^{\prime}\right) \subset \mathbb{R}^{d}, \quad \tilde{\mathcal{U}}^{\prime}:=\mathbf{p}\left(\mathcal{U}^{\prime}\right) \subset H \backslash G / B .
$$

The subsets $\mathcal{U}^{\prime}$ and $\mathcal{V}^{\prime}$ respectively of $G$ and $\mathbb{R}^{d}$ are Zariski open subsets and $\tilde{\mathcal{U}}^{\prime}$ is open and dense in $H \backslash G / B$. To simplify the notation, these subsets will be denoted respectively by $\mathcal{U}, \mathcal{V}$ and $\tilde{\mathcal{U}}$.

Remark 3.16. Since the representations $\tau_{\ell, \mathfrak{b}}$ and $\tau_{\mathrm{Ad}^{*}(g) \ell, \operatorname{Ad}(g) \mathfrak{b}}, g \in G$, are equivalent, we can replace the subalgebra $\mathfrak{b}$ by a conjugated algebra $\operatorname{Ad}(g) \mathfrak{b}$, with $g \in \mathcal{U}$. This allows us to assume, if we want, that $e$ is contained in the Zariski open subset $\mathcal{U}$. This means that we can suppose that 0 is an element of $\mathcal{V}$.

Theorem 3.17. The mapping

$$
\Phi: \mathbb{R}^{r} \times \mathcal{V} \longrightarrow G / B:(s, t) \mapsto E_{\mathcal{N}}(s) \phi(t) \cdot B
$$

is injective and polynomial, its image is equal to $\mathcal{U} \bmod B$ and it is regular on a Zariski open subset of $\mathbb{R}^{r} \times \mathcal{V}$.

Proof. Let $g \in \mathcal{U}$. Then $g=h \phi(t) b$ with $t \in \mathcal{V}, h \in H$ and $b \in B$. We can write $h=E_{\mathcal{N}}(s) h_{0}$, where $h_{0}=\phi(t) b_{0} \phi(t)^{-1}$ (with $b_{0} \in B$ ), is an element of $H \cap \phi(t) B \phi(t)^{-1}$. Hence,

$$
\Phi(s, t)=E_{\mathcal{N}}(s) \phi(t) \cdot B=E_{\mathcal{N}}(s) \phi(t) b_{0} \cdot B=E_{\mathcal{N}}(s) \phi(t) b_{0} \phi(t)^{-1} \phi(t) \cdot B=g \cdot B .
$$


If $\Phi(s, t)=\Phi\left(s^{\prime}, t^{\prime}\right)$ for two couples $(s, t)$ and $\left(s^{\prime}, t^{\prime}\right)$ in $\mathbb{R}^{r} \times \mathcal{V}$, then $H \cdot \phi(t) \cdot B=$ $H \cdot \phi\left(t^{\prime}\right) \cdot B$ and since $t \in \mathcal{V}$, it follows from the injectivity of $\tilde{\phi}$, that $t=t^{\prime}$. Hence $\ddot{E_{\mathcal{N}}}(s)=\ddot{E_{\mathcal{N}}}\left(s^{\prime}\right)$ and the injectivity of the mapping $\ddot{E_{\mathcal{N}}}$ implies that $s=s^{\prime}$.

Now, if we choose for instance the Malcev basis $\mathcal{M}=\left\{Z_{k_{1}}, \cdots, Z_{k_{p}}\right\}$ of $\mathfrak{g}$ relative to $\mathfrak{b}$ defined in (2.1), then for

$$
z=\exp \left(\sum_{j=1}^{n} z_{j} Z_{j}\right) \in G
$$

we have that

$$
z \bmod B=E_{\mathcal{U}}\left(Q_{1}\left(z_{1}, \cdots, z_{n}\right), \cdots, Q_{p}\left(z_{1}, \cdots, z_{n}\right)\right) \quad \bmod B,
$$

where $Q_{1}, \cdots, Q_{k}: \mathbb{R}^{n} \longrightarrow \mathbb{R}$ are real-valued polynomials. This tells us that $\Phi$ is a polynomial mapping, since the mapping $(s, t) \rightarrow E_{\mathcal{N}}(s) \phi(t)$ is clearly polynomial. The differential of the mapping $\Phi$ in zero is given by the family of vectors

$$
\left\{Y_{j_{1}}, \cdots, Y_{j_{r}}, Z_{i_{1}}, \cdots, Z_{i_{d}}\right\} \bmod \mathfrak{b} .
$$

These vectors form a basis of $\mathfrak{g}$ modulo $\mathfrak{b}$ (but not necessarily a Malcev basis). Hence $\Phi$ is regular in $(0,0)$ and since $\Phi$ is polynomial, $\Phi$ is regular on a Zariski open subset of $\mathbb{R}^{r} \times \mathbb{R}^{d}$.

Remark 3.18. As a consequence of the previous theorem, we have the following condition on the cardinals:

$$
\sharp \mathcal{I}^{\mathfrak{g} / \mathfrak{b}}=\sharp \mathcal{I}(\mathfrak{g} / \mathfrak{h}, \mathfrak{g} / \mathfrak{b})+\sharp \mathcal{J}^{\mathfrak{h} / \mathfrak{b}(G)},
$$

but in general,

$$
\mathcal{I}^{\mathfrak{g} / \mathfrak{b}} \neq \mathcal{I}(\mathfrak{g} / \mathfrak{h}, \mathfrak{g} / \mathfrak{b}) \cup \mathcal{J}^{\mathfrak{h} / \mathfrak{b}(G)} .
$$

This is shown by the following example.

Example 3.19. We use again Example 3.1. Clearly we have that $\mathcal{I}^{\mathfrak{g} / \mathfrak{b}}=\{1,2,5,6\}$ and we have seen that $\mathcal{I}(\mathfrak{g} / \mathfrak{h}, \mathfrak{g} / \mathfrak{b})=\{1,2\}$ and it follows easily that $\mathcal{J}^{\mathfrak{h} / \mathfrak{b}(G)}=$ $\{4,5\}$.

Corollary 3.20. We recall the definitions of the sets of indices.

- $\mathcal{I}(\mathfrak{g} / \mathfrak{h}, \mathfrak{g} / \mathfrak{b})=\left\{i_{1}, \cdots, i_{d}\right\}$ giving the directions $\left(Z_{i_{1}}, \cdots, Z_{i_{d}}\right)$ which characterize almost all elements of $H \backslash G / B$ by Theorem 3.10.

- $\mathcal{J}^{\mathfrak{h} / \mathfrak{b}(G)}=J=\left\{j_{1}, \cdots, j_{r}\right\}$ giving the Malcev basis $\mathcal{N}=\left(Y_{j_{1}}, \cdots, Y_{j_{r}}\right)$ of $\mathfrak{h}$ relative to $\mathfrak{b}(g)$ for every $g \in \mathcal{U}^{\prime \prime}$ defined in Notation 3.15 ,

- $\mathcal{I}^{\mathfrak{g} / \mathfrak{b}}=\left\{k_{1}, \cdots, k_{p}\right\}(p=r+d)$, giving the Malcev basis $\mathcal{M}=\left(Z_{k_{1}}, \cdots, Z_{k_{p}}\right)$ of $\mathfrak{g}$ relative to $\mathfrak{b}$ defined in (2.1).

As a consequence of Theorem 3.17, we have that the mapping

$$
\begin{gathered}
\Psi=\dot{E}_{\mathcal{M}}^{-1} \circ \Phi: \quad \begin{array}{c}
\mathbb{R}^{r} \times \mathcal{V} \\
\left(s=\left(s_{1}, \cdots, s_{r}\right), t=\left(t_{1}, \cdots, t_{d}\right)\right)
\end{array} \underset{\mathbb{R}^{p}}{\longmapsto} \zeta=\left(\zeta_{1}, \cdots, \zeta_{p}\right) \\
E_{\mathcal{N}}(s) \phi(t) \cdot B=s_{1} Y_{j_{1}} \ldots s_{r} Y_{j_{r}} \cdot t_{1} Z_{i_{1}} \ldots t_{d} Z_{i_{d}} \cdot B=\zeta_{1} Z_{k_{1}} \ldots \zeta_{p} Z_{k_{p}} \cdot B=E_{\mathcal{M}}(\zeta)
\end{gathered}
$$

is a polynomial diffeomorphism into its image $\mathcal{O}$, which is a Zariski open subset of $\mathbb{R}^{p}$. 
Notation 3.21. For $t \in \mathcal{V}$, we denote by $E_{t}$ the diffeomorphism between $H$ and $\mathbb{R}^{r} \times B(t)$ such that for $h \in H$, and $(s, b) \in \mathbb{R}^{r} \times B(t)$,

$$
E_{t}(h)=(s, b) \quad \Longleftrightarrow \quad h=E_{\mathcal{N}}(s) b .
$$

Write also for $t \in \mathcal{V}$ and $h \in H$, the element $s(h, t)$ of $\mathbb{R}^{r}$ satisfying

$$
h=E_{\mathcal{N}}(s(h, t)) \bmod B(t) .
$$

We now fix $t \in \mathcal{V}$ and $h \in H$ in order to define the polynomial mapping $q_{h, t}$ : $\mathbb{R}^{r} \longrightarrow \mathbb{R}^{r}$ by

$$
q_{h, t}(s)=\ddot{E}_{\mathcal{N}}^{-1}\left(h E_{\mathcal{N}}(s)\right)=s\left(h E_{\mathcal{N}}(s), t\right), s \in \mathbb{R}^{r} .
$$

It follows then that

$$
q_{h h^{\prime}, t}=q_{h, t} \circ q_{h^{\prime}, t}, h, h^{\prime} \in H, t \in \mathcal{V} .
$$

Proposition 3.22. The Jacobian Jac $(s, t)$ of the mapping $\Phi$ of Theorem 3.17 is constant in $s \in \mathbb{R}^{r}$.

Proof. Indeed, if $f \in C_{c}(\mathcal{U} / B), t \in \mathcal{V}$, and $h^{\prime} \in H$, using the left-invariant measure $d \dot{g}$ on $G / B$, we get

$$
\begin{aligned}
\int_{\mathbb{R}^{d}} \int_{\mathbb{R}^{r}} f(\Phi(s, t))|J a c(s, t)| d s d t & =\int_{G / B} f(g) d \dot{g}=\int_{G / B} f\left(h^{\prime} g\right) d \dot{g} \\
& =\int_{\mathbb{R}^{d}} \int_{H / B(t)} f\left(h^{\prime} h \phi(t)\right)|J a c(s((h, t), t))| d_{\mathcal{N}}(h) d t \\
& =\int_{\mathbb{R}^{d}} \int_{H / B(t)} f(h \phi(t))\left|J a c\left(s\left(h^{\prime-1} h, t\right), t\right)\right| d \ddot{h} d t \\
& =\int_{\mathbb{R}^{d}} \int_{\mathbb{R}^{r}} f\left(E_{\mathcal{N}}(s) \phi(t)\right)\left|J a c\left(q_{h^{\prime-1}, t}(s), t\right)\right| d s d t \\
& =\int_{\mathbb{R}^{d}} \int_{\mathbb{R}^{r}} f(\Phi(s, t))\left|J a c\left(q_{h^{\prime-1}, t}(s), t\right)\right| d s d t .
\end{aligned}
$$

This shows that $\left|\operatorname{Jac}\left(q_{h^{\prime-1}, t}(s), t\right)\right|=|\operatorname{Jac}(s, t)|$ for every $h^{\prime} \in H, s \in \mathbb{R}^{r}$ and $t \in \mathcal{V}$. Therefore $\operatorname{Jac}(s, t)=\operatorname{Jac}(0, t)$ for every $s \in \mathbb{R}^{r}, t \in \mathcal{V}$, since $\Phi$ is a real-valued polynomial function.

Definition 3.23. Let

$$
F(t):=\operatorname{Jac}(0, t), t \in \mathcal{V} .
$$

Then $F: \mathcal{V} \longrightarrow \mathbb{R}$ is a function on $\mathcal{V}$ which admits a polynomial extension on $\mathbb{R}^{d}$.

Definition 3.24. We can define now our measure on the topological space $H \backslash G / B$. We have the Zariski dense open subset $\mathcal{U}$ of $G$, which has property $(\mathcal{P})$ and therefore its complement $\mathcal{C}$ in $G$ is Zariski closed and has also property $(\mathcal{P})$. The projection $\mathbf{p}: G \longrightarrow H \backslash G / B$ maps $\mathcal{U}$ to the open dense subset $\tilde{\mathcal{U}}$ and $\mathcal{C}$ to the closed subset $\tilde{\mathcal{C}}$. We define a measure $d \gamma$, which is supported on $\tilde{\mathcal{U}}$, by the rule

$$
\int_{H \backslash G / B} f(x) d \gamma(x):=\int_{\mathcal{V}} f(\phi(t))|F(t)| d t .
$$

Proposition 3.25. Let $f \in C_{c}(G / B)$. Then

$$
\int_{G / B} f(g) d \dot{g}=\int_{H \backslash G / B}\left(\int_{H / B(x)} f(h \psi(x)) d \ddot{h}\right) d \gamma(x) .
$$


Proof. We can make the following computations:

$$
\begin{aligned}
\int_{G / B} f(g) d \dot{g} & =\int_{\mathbb{R}^{d}} \int_{\mathbb{R}^{r}} f(\Phi(s, t))|J a c(s, t)| d s d t \\
& =\int_{\mathcal{V}}\left(\int_{\mathbb{R}^{r}} f\left(E_{\mathcal{N}}(s) \phi(t)\right) d s\right)|F(t)| d t \\
& =\int_{\mathcal{V}}\left(\int_{H / B(t)} f(h \phi(t)) d_{\mathcal{N}}(h)\right)|F(t)| d t
\end{aligned}
$$

and justify them by

- Theorem 3.17 for the first equality,

- Proposition 3.22 and Definition 3.23 for the second one,

- Reference (3.7) for the last statement; in fact $\mathcal{N}$ is a Malcev basis of $\mathfrak{h}$ relative to all the $\mathfrak{b}(t), t \in \mathcal{V}$.

We finally conclude by Definition 3.24 .

Notation 3.26. Let $H=\exp (\mathfrak{h})$ be a closed subgroup of the simply connected nilpotent Lie group $G=\exp (\mathfrak{g})$. Let $\ell \in \mathfrak{g}^{*}$, let $B=\exp (\mathfrak{b})$ be a polarization at $\ell$ and $\pi_{\ell}=\pi_{\ell, \mathfrak{b}}=\operatorname{ind}_{B}^{G} \chi_{\ell}$ the irreducible representation associated to $(\ell, \mathfrak{b})$. Let for $g \in \mathcal{U}$

$$
\ell(g)=\operatorname{Ad}^{*}(g) \ell_{\mid \mathfrak{h}} \quad \text { and } \quad \tau_{\ell(g), \mathfrak{b}(g)}=\operatorname{ind}_{B(g)}^{H} \chi_{\ell(g)}=: \sigma(g) .
$$

Then we can show that the representations $\sigma\left(g^{\prime}\right)$ and $\sigma(g)$ of $H$ are equivalent whenever $g^{\prime}$ and $g$ are "equivalent", i.e. whenever $g$ and $g^{\prime}$ are in the same double coset. Hence, when $\tilde{g}=\tilde{\phi}(t) \in \tilde{\mathcal{U}}$, we define $\sigma(\tilde{g})=\sigma(t):=[\sigma(g)]$ as the equivalence class of the unitary representation $\sigma(g)$.

Proposition 3.27. The restriction of $\pi_{\ell, \mathfrak{b}}$ to $H$ is a direct integral over the double cosets space $H \backslash G / B$ (with respect to the measure $d \gamma$ ) and the contribution of $x \in \tilde{\mathcal{U}} \subset H \backslash G / B$ may be computed as follows. Recall (3.5) where $\ell(x) \in \mathfrak{h}^{*}$ is the character $\ell(\psi(x))$ on the closed subgroup $B(x)=\exp (\mathfrak{b}(x))$ of $H$. Hence, the isotopic components in the disintegration of $\pi_{\ell, \mathfrak{b}}$ to $H$ are the representations $\sigma(x)=\tau_{\ell(x), \mathfrak{b}(x)}$ whose Hilbert spaces are $\mathcal{H}_{\sigma(x)}=L^{2}\left(H / B(x), \chi_{\ell(x)}\right)$. We have

$$
L^{2}\left(G / B, \chi_{\ell}\right) \simeq \int_{H \backslash G / B}^{\oplus} \mathcal{H}_{\sigma(x)} d \gamma(x)
$$

and

$$
\pi_{\ell, \mathfrak{b}_{\mid H}} \simeq \int_{H \backslash G / B}^{\oplus} \sigma(x) d \gamma(x) .
$$

Proof. Let $L_{0}$ be the space of all the vector fields $F=\left(f_{x} \in C_{c}(H / B(x))\right)_{x \in \tilde{\mathcal{U}}}$, such that the function $\mathbb{R}^{r} \times \tilde{\mathcal{U}} \ni(s, x) \mapsto f_{x}\left(E_{\mathcal{N}}(s)\right)$ is continuous with compact support. On $L_{0}$ we have the Hilbert space norm

$$
\|F\|_{2}^{2}:=\int_{H \backslash G / B} \int_{\mathbb{R}^{r}}\left|F_{x}\left(E_{\mathcal{N}}(s)\right)\right|^{2} d s d \gamma(x)=\int_{H \backslash G / B}\left\|F_{x}\right\|_{L^{2}\left(H / B(x), \chi_{\ell(x)}\right)}^{2} d \gamma(x) .
$$

We construct now an isometric isomorphism $S$ between $L^{2}\left(G / B, \chi_{\ell}\right)$ and the direct integral

$$
\int_{H \backslash G / B}^{\oplus} L^{2}\left(H / B(x), \chi_{\ell(x)}\right) d \gamma(x),
$$

which can be defined as the completion of $L_{0}$ for the norm \|\|$_{2}$ of $L_{0}$. 
For $\xi \in C_{c}\left(G / B, \chi_{\ell}\right)$, define the function

$$
S \xi(x)(h):=\xi(h \psi(x)), x \in \tilde{\mathcal{U}}, h \in H .
$$

For $x \in \tilde{\mathcal{U}}$, the function $S(\xi)(x)$ is then an element of $C_{c}\left(H / B(x), \chi_{\ell(x)}\right)$, since it satisfies the covariance relation

$$
S(\xi)(x)(h b)=\xi(h b \phi(t))=\xi\left(h \phi(t) \phi(t)^{-1} b \phi(t)\right)=\chi_{\ell(x)} \xi(h \phi(t))=\chi_{\ell(x)} S(\xi)(x)(h),
$$

where $\phi(t)=\psi(x), h \in H$, and $b \in B(x)$. It is also continuous with compact support modulo $B(x)$ for every $x \in \tilde{\mathcal{U}}$, since the mapping $\Phi: \mathbb{R}^{r} \times \mathcal{V} \longrightarrow \mathcal{U}$ is a homeomorphism.

We obtain in this way a vector field $\left(S(\xi)(x) \in C_{c}\left(H / B(x), \chi_{\ell(x)}\right)\right)_{x \in \tilde{\mathcal{U}}}$, which is contained in $L_{0}$. The mapping $S: C_{c}\left(G / B, \chi_{\ell}\right) \rightarrow L_{0}$ is isometric by Proposition 3.25 it is a linear isomorphism and it obviously intertwines the representation $\pi_{\ell \mid H}$ and the direct integral representation

$$
\int_{H \backslash G / B}^{\oplus} \tau_{\chi \ell(x), B(x)} d \gamma(x) .
$$

\section{Disintegration of induced Representations}

We have seen in the preceding section that the disintegration of the restriction of the irreducible representation $\pi_{\ell, \mathfrak{b}}$ to $H$ reduces now to the disintegration of the monomial representations $\sigma(x)=\tau_{\chi_{\ell}(x), B(x)}$ of $H$ into irreducibles. We shall use the techniques developed in [2].

In Notation 3.15, we introduced the Malcev basis $\mathcal{N}=\left\{Y_{j_{1}}, \cdots, Y_{j_{r}}\right\}$ of $\mathfrak{h}$ relative to all the $\mathfrak{b}(g), g \in \mathcal{U}$. Let us use also the following notation.

Notation 4.1. For every $t \in \mathcal{V}$,

- we have similarly to (2.1) a flag of subalgebras

$$
\mathfrak{b}^{r+1}(t):=\mathfrak{b}(t) \subset \cdots \subset \mathfrak{b}^{c}(t)=\operatorname{span}\left\langle Y_{j_{c}}, \cdots, Y_{j_{r}}, \mathfrak{b}(t)\right\rangle \subset \cdots \subset \mathfrak{b}^{1}(t)=\mathfrak{h} .
$$

We denote their Lie groups by $B^{c}(t):=\exp \mathfrak{b}^{c}(t), c=1, \cdots, r$.

- Let $\mathcal{X}(t)$ be a Jordan-Hölder basis of $\mathfrak{b}(t)$ generated by rationally varied vectors $X_{i}(t), i=r+1, \cdots, m$, with no singularities in $t \in \mathcal{V}$. The corresponding Malcev basis of $\mathfrak{h}$ is then

$$
\mathcal{Y}(t)=\left\{X_{1}(t):=Y_{j_{1}}, \cdots, X_{r}(t):=Y_{j_{r}}, X_{r+1}(t), \cdots, X_{m}(t)\right\},
$$

and has the dual basis in $\mathfrak{h}^{*}$

$$
\mathcal{Y}(t)^{*}=\left\{X_{1}(t)^{*}, \cdots, X_{r}(t)^{*}, X_{r+1}(t)^{*}, \cdots, X_{m}(t)^{*}\right\}, \quad t \in \mathcal{V} .
$$

- Let

$$
\Gamma(t)=\ell(t)+\mathfrak{b}(t)^{\perp} \quad \text { where } \quad \ell(t):=\operatorname{Ad}^{*}(\phi(t)) \ell_{\mid \mathfrak{h}} .
$$

Also let $d_{c}(t):=\max \left\{\operatorname{dim}\left(\operatorname{Ad}^{*}\left(B^{c}(t)\right)(f)\right) ; f \in \Gamma(t)\right\}$ for any $1 \leq c \leq r$ and put by convention $d_{r+1}(t):=0$. We obtain then an index set

$$
I(t):=\left\{c_{1}<\cdots<c_{q(t)}\right\}:=\left\{c \in\{1, \cdots, r\}, \quad d_{c}(t)=d_{c+1}(t)\right\}
$$

and an affine space, on which we take the Lebesgue measure $d v$,

$$
\mathcal{R}(t):=\left\{\ell(t)+\sum_{c_{i} \in I(t)} v_{i} X_{c_{i}}(t)^{*}, \quad v=\left(v_{1}, \cdots, v_{q(t)}\right) \in \mathbb{R}^{q(t)}\right\} .
$$


We can again assume, if necessary by shrinking $\mathcal{V}$, that there exists a fixed index set $I$ such that for every $t \in \mathcal{V}$ we have that

$$
I(t)=I=\left\{c_{1}, \cdots, c_{q}\right\} \text { and so } q(t)=q=|I| .
$$

- Denote now by

$$
\ell(t, v):=\ell(t)+\sum_{c \in I(t)} v_{i} Y_{j_{c}}(t)^{*} \in \Gamma(t), v \in \mathbb{R}^{q}
$$

and by $\mathfrak{k}(t, v)$ the Vergne polarization of $\mathfrak{h}$ at $\ell(t, v)$ relative to the JordanHölder basis

$$
\mathcal{Y}=\left\{Y_{1}, \cdots, Y_{m}\right\}=\left\{Z_{j}, j \in \mathcal{I}^{\mathfrak{h}}\right\} .
$$

We denote by $\rho_{(t, v)}=\rho_{w}$ the unitary irreducible representation of $H$,

$$
\rho_{f}=\operatorname{ind}_{K(t, v)}^{H} \chi_{f} \in \hat{H},
$$

for any $f=f_{w}=\ell(t, v) \in \mathcal{R}(t)$ where $w=(t, v), v \in \mathbb{R}^{q}$ and $K(t, v)=$ $\exp (\mathfrak{k}(t, v))$.

- Finally, we identify any $x \in \tilde{\mathcal{U}} \subset H \backslash G / B$ with $\theta(x) \in \mathbb{R}^{d}$; in particular when $t \in \mathcal{V}$ and $x=\tilde{\phi}(t)$, then we put $\mathcal{R}(x):=\mathcal{R}(t)$. It follows that if $v \in \mathcal{R}(x)$, then $(x, v):=(t, v), \rho_{(x, v)}:=\rho_{(t, v)}, K(x, v):=K(t, v)$, etc $\ldots$

It follows from the proofs used in the paper [2 that there exists a Zariski open subset $\mathcal{W}$ of $\mathbb{R}^{d} \times \mathbb{R}^{q}$ contained in $\mathcal{V} \times \mathbb{R}^{q}$ such that for every $w=(t, v) \in \mathcal{W}$, we have a Malcev basis

$$
\mathcal{X}(w)=\left\{X_{1}(w), \cdots, X_{d}(w)\right\}
$$

of $\mathfrak{h}$ relative to $\mathfrak{k}(t, v)$ and a Malcev basis

$$
\mathcal{Y}(w)=\left\{Y_{1}(w), \cdots, Y_{e}(w)\right\}
$$

of $\mathfrak{k}(t, v)$ relative to the subalgebra $\mathfrak{k}(t, v) \cap \mathfrak{b}(t)$ such that all the vectors $X_{j}(w)$ and $Y_{i}(w)$ vary rationally without singularities in $w=(t, v) \in \mathcal{W}$. Define now the operators

$$
T_{w}: L^{2}\left(H / B(t), \chi_{\ell(t)}\right) \longrightarrow L^{2}\left(H / K(t, v), \chi_{\ell(w)}\right), \quad w=(t, v) \in \mathcal{W}
$$

such that

$$
T_{w}(\eta)(h)=\int_{K(t, v) / K(t, v) \cap B(t)} \eta(h b) \chi_{\ell(w)}(b) d_{\mathcal{Y}(w)}(b), \eta \in L^{2}\left(H / B(t), \chi_{\ell(t)}\right), h \in H
$$

and where the measure $d_{\mathcal{Y}(w)}$ on $K(w) /(K(w) \cap B(t))$ is built using the Malcev basis $\mathcal{Y}(w)$.

Thus, for a fixed $t \in \mathcal{V}$, we obtain an isometric intertwining operator

$$
\mathcal{T}^{t}: L^{2}\left(H / B(t), \chi_{\ell(t)}\right) \longrightarrow \int_{\mathcal{R}(t)}^{\oplus} L^{2}\left(H / K(t, v), \chi_{\ell(t, v)}\right) d v
$$

characterized by the following properties.

(1) It takes any $\eta \in L^{2}\left(H / B(t), \chi_{\ell(t)}\right)$ to

$$
\mathcal{T}^{t}(\eta): \mathcal{R}(t) \rightarrow \bigcup_{\mathcal{R}(t)} L^{2}\left(H / K(t, v), \chi_{\ell(t, v)}\right)
$$


such that for $f_{w} \in \mathcal{R}(t)$ with $w=(t, v) \in \mathcal{W},\left(\mathcal{T}^{t} \eta\right)\left(f_{w}\right):=T_{w}(\eta) \in$ $L^{2}\left(H / K(w), \chi_{\ell(w)}\right)$. Notice that the norm on this space is defined via the measure $d_{\mathcal{X}(w)}$ on $H / K(w)$.

(2) It intertwines the monomial representation

$$
\sigma(t)=\operatorname{ind}_{B(t)}^{H} \chi_{\ell(t)}
$$

with its disintegration into irreducibles of $H$ given by

$$
\int_{\mathcal{R}(t)}^{\oplus} \rho_{(t, v)} d v=\int_{\mathcal{R}(t)}^{\oplus} \operatorname{ind}_{K(t, v)}^{H} \chi_{\ell(t, v)} d v .
$$

Combining these manipulations with Theorem 3.3 of 2$]$ and with Proposition 3.27 we obtain Theorem 4.2 giving an isometric intertwining operator

$$
\mathcal{T}: L^{2}\left(G / B, \chi_{\ell}\right) \longrightarrow \int_{\mathcal{V}}^{\oplus}\left(\int_{\mathcal{R}(t)}^{\oplus} L^{2}\left(H / K(t, v), \chi_{\ell(t, v)}\right) d v\right)|F(t)| d t
$$

between $\pi_{\ell \mid H}$ and its decomposition into irreducibles of $H$,

$$
\pi_{\ell, \mathfrak{b}_{\mid H}} \simeq \int_{H \backslash G / B}^{\oplus}\left(\int_{\mathcal{R}(x)}^{\oplus} \rho_{(x, v)} d v\right) d \gamma(x) \simeq \int_{\mathcal{W}}^{\oplus} \rho_{w} d w,
$$

where the measure $d w$ that we take on $\mathcal{W}$ is $|F(t)| d t \otimes d v$ on $\mathbb{R}^{d} \times \mathbb{R}^{q}$. Let's recall the operator $S$ introduced in the proof of Proposition 3.27 whose expression

$$
\begin{aligned}
& S(\xi) \in \int_{H \backslash G / B}^{\oplus} L^{2}\left(H / B(x), \chi_{\ell(x)}\right) d \gamma(x): \\
& H \backslash G / B \ni x \mapsto S \xi(x) \in L^{2}\left(H / B(x), \chi_{\ell(x)}\right)
\end{aligned}
$$

is given by (3.8) $\forall h \in H, S \xi(x)(h)=\xi(h \psi(x))$ for every $\xi \in \mathcal{S}\left(G / B, \chi_{\ell}\right)$. So define

$$
\begin{aligned}
\mathcal{T}(\xi): \mathcal{V} \ni t \mapsto \mathcal{T} \xi(t)= & \mathcal{T}^{t}(S \xi(\tilde{\phi}(t))) \quad \in \int_{\mathcal{R}(t)}^{\oplus} L^{2}\left(H / K(t, v), \chi_{\ell(t, v)}\right) d v, \\
\mathcal{R}(t) \ni f_{(t, v)} & \mapsto T_{(t, v)}(S \xi(\tilde{\phi}(t))) \in L^{2}\left(H / K(t, v), \chi_{\ell(t, v)}\right) .
\end{aligned}
$$

Then, for every $f_{w}=\ell(t, v) \in \mathcal{R}(x)$ where $w=(t, v) \in \mathcal{W}$, we see that

$$
\begin{aligned}
\mathcal{T} \xi(w) & :=\mathcal{T} \xi\left(x, f_{w}\right)=T_{w}(S \xi(x)) \in L^{2}\left(H / K(w), \chi_{\ell(w)}\right) \\
H \ni h & \mapsto \int_{K(t, v) /(K(t, v) \cap B(t))} \xi(h b \phi(t)) \chi_{\ell(w)}(b) d_{\mathcal{Y}(w)}(b) \in \mathbb{C} .
\end{aligned}
$$

Theorem 4.2. Let $G=\exp \mathfrak{g}$ be a connected simply connected nilpotent Lie group and $H$ a closed connected subgroup of $G$. Let $\mathcal{Z}$ be a Jordan-Hölder basis of $\mathfrak{g}, \ell$ in $\mathfrak{g}^{*}$ and $\mathfrak{b}$ the Vergne polarization at $\ell$ with respect to $\mathcal{Z}$. Also let $\pi$ be an irreducible representation of $G$ realized as $\pi_{\ell, \mathfrak{b}}=\operatorname{ind}_{B}^{G} \chi_{\ell}$. Then the operator $\mathcal{T}$ defined on $\mathcal{S}\left(G / B, \chi_{\ell}\right)$ as in Reference (4.10) uniquely extends to a unitary intertwining operator which decomposes the restriction of $\pi$ to $H$ as in Reference (4.9).

Remark 4.3. Let's remark that for any $\xi \in \mathcal{S}\left(G / B, \chi_{\ell}\right)$, one has

$$
\begin{aligned}
\|\xi\|_{L^{2}\left(G / B, \chi_{\ell}\right)}^{2} & =\int_{H \backslash G / B}\left(\int_{\mathcal{R}(x)}\left\|T_{(x, v)} S \xi(x)\right\|_{L^{2}\left(H / K(x, v) ; \chi_{\ell(x, v)}\right)}^{2} d v\right) d \gamma(x) \\
& =\int_{\mathcal{W}}\|\mathcal{T} \xi(w)\|_{L^{2}\left(H / K(w) ; \chi_{f_{w}}\right)}^{2} d w .
\end{aligned}
$$




\section{Disintegrating TENSOR PRODUCTS OF IRREDUCIBLE REPRESENTATIONS}

Let $G=\exp \mathfrak{g}$ be a simply connected, connected Lie group and let $\pi=\pi_{\ell}, \pi^{\prime}=$ $\pi_{\ell^{\prime}}$ be two irreducible unitary representations of $G$. We take a Jordan-Hölder basis $\mathcal{Z}=\left\{Z_{1}, \cdots, Z_{n}\right\}$ of $\mathfrak{g}$ and we obtain the corresponding Vergne polarizations $\mathfrak{b}$ at $\ell$ and $\mathfrak{b}^{\prime}$ at $\ell^{\prime}$. We then realize $\pi$ as $\pi_{\ell, \mathfrak{b}}$ and $\pi^{\prime}$ as $\pi_{\ell^{\prime}, \mathfrak{b}^{\prime}}$.

Let $\mathbb{G}:=G \times G$ and $\mathbb{B}:=B \times B^{\prime}$ be the direct products respectively of $G$ with $G$ and $B$ with $B^{\prime}$. We denote by $\mathcal{G}:=\mathfrak{g} \times \mathfrak{g}$ and $\mathcal{B}:=\mathfrak{b} \times b^{\prime}$ their respective Lie algebras. Also let $\boldsymbol{\Pi}=\pi \times \pi^{\prime}$ be the outer tensor product of $\pi$ and $\pi^{\prime}$. We can realize $\Pi$ as an induced representation. It acts by left translation on the space

$$
\mathcal{H}_{\Pi}:=L^{2}\left(\mathbb{G} / \mathbb{B}, \chi_{\left(\ell, \ell^{\prime}\right)}\right) \simeq \mathcal{H}_{\pi} \hat{\otimes} \mathcal{H}_{\pi^{\prime}},
$$

where $\left(\ell, \ell^{\prime}\right)$ is the linear functional defined on $\mathfrak{g} \times \mathfrak{g}$ by

$$
\left(\ell, \ell^{\prime}\right)\left(Z, Z^{\prime}\right):=\ell(Z)+\ell^{\prime}\left(Z^{\prime}\right), Z, Z^{\prime} \in \mathfrak{g},
$$

and so $\mathcal{B}$ is a polarization at $\left(\ell, \ell^{\prime}\right)$. Hence $\Pi=\pi_{\left(\ell, \ell^{\prime}\right), \mathcal{B}}$.

Let $\mathbb{D}=\{(g, g) ; g \in G\}$ be the diagonal group of $\mathbb{G}$. This group is of course isomorphic to $G$ and the tensor product $\pi \otimes \pi^{\prime}$ is the representation of $G$ acting on the space of $\Pi$ through the rule

$$
\pi \otimes \pi^{\prime}(g):=\Pi(g, g), g \in G .
$$

We are led to investigate the structure of the space of double cosets $\mathbb{D} \backslash \mathbb{G} / \mathbb{B}$.

Proposition 5.1. The mapping

$$
\mathbb{M}: B \backslash G / B^{\prime} \longrightarrow \mathbb{D} \backslash \mathbb{G} / \mathbb{B}, B \cdot g \cdot B^{\prime} \mapsto \mathbb{D} \cdot(e, g) \cdot \mathbb{B},
$$

is well defined and is a bijection.

Proof. Indeed, if $g \in G$ and $g^{\prime} \in B \cdot g \cdot B^{\prime}$, then we have that $g^{\prime}=b g b^{\prime}$ for some $b \in B$ and $b^{\prime} \in B^{\prime}$ and so

$$
\begin{aligned}
\mathbb{D} \cdot\left(e, g^{\prime}\right) \cdot \mathbb{B} & =\mathbb{D} \cdot\left(e, b g b^{\prime}\right) \cdot \mathbb{B}=\left\{\left(u b_{2}, u b g b^{\prime} b_{2}^{\prime}\right), u \in G, b_{2} \in B, b_{2}^{\prime} \in B^{\prime}\right\} \\
& =\left\{\left(u b^{-1} b_{2}, u g b_{2}^{\prime}\right), u \in G, b_{2} \in B, b_{2}^{\prime} \in B^{\prime}\right\} \\
& =\left\{\left(u b_{2}, u g b_{2}^{\prime}\right), u \in G, b_{2} \in B, b_{2}^{\prime} \in B^{\prime}\right\} \\
& =\mathbb{D} \cdot(e, g) \cdot \mathbb{B} .
\end{aligned}
$$

Hence our mapping is well defined. Since

$$
\mathbb{D} \cdot\left(g, g^{\prime}\right) \cdot \mathbb{B}=\mathbb{D} \cdot(g, g) \cdot\left(e, g^{-1} g^{\prime}\right) \cdot \mathbb{B}=\mathbb{D} \cdot\left(e, g^{-1} g^{\prime}\right) \cdot \mathbb{B}=\mathbb{M}\left(B \cdot\left(g^{-1} g^{\prime}\right) \cdot B^{\prime}\right),
$$

it is also surjective. If $\mathbb{M}\left(B \cdot g \cdot B^{\prime}\right)=\mathbb{M}\left(B \cdot g^{\prime} \cdot B^{\prime}\right)$ for two elements $g, g^{\prime}$ in $G$, then $\left(e, g^{\prime}\right)=(u, u)(e, g)\left(b, b^{\prime}\right)$ for some $u \in G, b \in B$ and $b^{\prime} \in B^{\prime}$. Hence, $u b=e$ and $u g b^{\prime}=g^{\prime}$, which means that $g^{\prime}=b^{-1} g b^{\prime}$ is an element of $B \cdot g \cdot B^{\prime}$. Therefore $\mathbb{M}$ is injective and so it is a bijection.

According to the two preceding sections, we first need a Jordan-Hölder basis of $\mathfrak{g} \times \mathfrak{g}$ in order to perform our computations.

We make the following choice. Let

$$
\mathcal{Z}_{i}:=\left\{\begin{array}{cl}
\left(Z_{j}, Z_{j}\right) & \text { if } i=2 j-1 \text { is odd, } \\
\left(Z_{j},-Z_{j}\right) & \text { if } i=2 j \text { is even }
\end{array} \quad(j \in\{1, \cdots, n\}) .\right.
$$

The basis $\mathcal{A}=\left\{A_{1}, \cdots, A_{n}\right\}$ of the Lie algebra $\mathfrak{d}=\{(Z, Z) ; Z \in \mathfrak{g}\}$ of the diagonal group $\mathbb{D}$ is then given by the vectors

$$
A_{j}:=\left(Z_{j}, Z_{j}\right)=\mathcal{Z}_{2 j-1}, \quad j=1, \cdots, n .
$$


Hence

$$
\mathcal{I}^{\mathfrak{d}}=\{2 j-1, j \in\{1, \cdots, n\}\}, \mathcal{I}^{\mathcal{G} / \mathfrak{d}}=\{2 j, j \in\{1, \cdots, n\}\} .
$$

We must determine the index sets $\mathcal{I}(\mathcal{G} / \mathfrak{d}, \mathcal{G} / \mathcal{B}) \subset \mathcal{I}^{\mathcal{G}} / \mathfrak{o} \cap \mathcal{I}^{\mathcal{G}} / \mathcal{B}$. By (5.1) we have that

$$
\mathcal{I}(\mathcal{G} / \mathfrak{d}, \mathcal{G} / \mathcal{B}) \subset\{2 j, j \in\{1, \cdots, n\}\} .
$$

Proposition 5.2.

$$
\mathcal{I}(\mathcal{G} / \mathfrak{d}, \mathcal{G} / \mathcal{B})=\left\{2 j, j \in \mathcal{I}\left(\mathfrak{g} / \mathfrak{b}, \mathfrak{g} / \mathfrak{b}^{\prime}\right)\right\} .
$$

Proof. Let $\mathcal{U}$ be a Zariski open subset of $G$ with property $(\mathcal{P})$, such that for every $j \in \mathcal{I}\left(\mathfrak{g} / \mathfrak{b}, \mathfrak{g} / \mathfrak{b}^{\prime}\right)$,

$$
Z_{j} \notin \mathfrak{b}+\operatorname{Ad}(g) \mathfrak{b}^{\prime}+\mathfrak{g}_{j+1} \text { for every } g \in \mathcal{U}
$$

Let

$$
\mathbb{U}:=\left\{\left(g, g^{\prime}\right) \in \mathbb{G}, g^{-1} g^{\prime} \in \mathcal{U}\right\} .
$$

Then $\mathbb{U}$ is a Zariski open subset of $\mathbb{G}$. Let us show that for every $j \in \mathcal{I}\left(\mathfrak{g} / \mathfrak{b}, \mathfrak{g} / \mathfrak{b}^{\prime}\right)$, we have

$$
\mathcal{Z}_{2 j}=\left(Z_{j},-Z_{j}\right) \notin \mathfrak{d}+\operatorname{Ad}\left(g, g^{\prime}\right)(\mathcal{B})+\left(\mathfrak{g} \times \mathfrak{g}^{\prime}\right)_{2 j+1}, \forall\left(g, g^{\prime}\right) \in \mathbb{U} .
$$

Then necessarily the index $2 j$ is in $\mathcal{I}(\mathcal{G} / \mathfrak{d}, \mathcal{G} / \mathcal{B})$. Suppose that for some elements $j$ and $\left(g, g^{\prime}\right)$ respectively of $\mathcal{I}\left(\mathfrak{g} / \mathfrak{b}, \mathfrak{g} / \mathfrak{b}^{\prime}\right)$ and $\mathbb{U}$, the relation (5.2) is false. Then we have that

$$
\left(Z_{j},-Z_{j}\right)=(Y, Y)+\left(\operatorname{Ad}(g)(X), \operatorname{Ad}\left(g^{\prime}\right)\left(X^{\prime}\right)\right)+\left(U, U^{\prime}\right)
$$

for certain vectors $Y, X, X^{\prime}$ in $\mathfrak{g}, \mathfrak{b}, \mathfrak{b}^{\prime}$ respectively and $U$ and $U^{\prime} \in \mathfrak{g}_{j+1}$, since $\left(\mathfrak{g} \times \mathfrak{g}^{\prime}\right)_{2 j+1}=\mathfrak{g}_{j+1} \times \mathfrak{g}_{j+1}$.

Hence

$$
\left\{\begin{array}{ccc}
Z_{j} & = & Y+\operatorname{Ad}(g)(X)+U \\
-Z_{j} & = & Y+\operatorname{Ad}\left(g^{\prime}\right)\left(X^{\prime}\right)+U^{\prime}
\end{array}\right.
$$

which gives us the identity

$$
Z_{j}=\operatorname{Ad}(g)\left(\frac{X}{2}\right)+\operatorname{Ad}\left(g^{\prime}\right)\left(\frac{-X^{\prime}}{2}\right)+\frac{U-U^{\prime}}{2} .
$$

This implies that

$$
\begin{array}{rlr}
Z_{j} & \equiv \frac{X}{2}+\operatorname{Ad}\left(g^{-1} g^{\prime}\right)\left(\frac{-X^{\prime}}{2}\right) & \bmod \mathfrak{g}_{j+1} \\
& \in \mathfrak{b}+\operatorname{Ad}\left(g^{-1} g^{\prime}\right)\left(\mathfrak{b}^{\prime}\right) & \bmod \mathfrak{g}_{j+1},
\end{array}
$$

where $g^{-1} g^{\prime} \in \mathcal{U}$ (by the definition of $\mathbb{U}$ ). This relation contradicts the fact that $j \in \mathcal{I}\left(\mathfrak{g} / \mathfrak{b}, \mathfrak{g} / \mathfrak{b}^{\prime}\right)$.

Now let $2 j \in \mathcal{I}(\mathcal{G} / \mathfrak{d}, \mathcal{G} / \mathcal{B})$. Then for every $\left(g, g^{\prime}\right)$ in a Zariski open subset of $\mathbb{G}$, we have that

$$
\left(Z_{j},-Z_{j}\right) \notin \mathfrak{d}+\operatorname{Ad}(g)(\mathfrak{b}) \times \operatorname{Ad}\left(g^{\prime}\right)\left(\mathfrak{b}^{\prime}\right)+\mathfrak{g}_{j+1} \times \mathfrak{g}_{j+1} .
$$


Suppose now that $j \notin \mathcal{I}\left(\mathfrak{g} / \mathfrak{b}, \mathfrak{g} / \mathfrak{b}^{\prime}\right)$. Then in the Zariski open subset $\mathcal{U}$ of $G$, we have that

$$
Z_{j} \in \mathfrak{b}+\operatorname{Ad}(g) \mathfrak{b}^{\prime}+\mathfrak{g}_{j+1}, \quad \forall g \in \mathcal{U}
$$

Let $\left(g, g^{\prime}\right)$ be one element of the Zariski open subset $\mathbb{U}$ of $\mathbb{G}$. This means that $g^{\prime \prime}:=g^{-1} g^{\prime} \in \mathcal{U}$. There exists $X \in \mathfrak{b}$ and $X^{\prime} \in \mathfrak{b}^{\prime}$, such that

$$
Z_{j}=X+\operatorname{Ad}\left(g^{\prime \prime}\right)\left(X^{\prime}\right) \bmod \mathfrak{g}_{j+1} .
$$

Hence

$$
\operatorname{Ad}(g) Z_{j}=\operatorname{Ad}(g) X+\operatorname{Ad}\left(g^{\prime}\right) X^{\prime} \bmod \mathfrak{g}_{j+1}
$$

and $Z_{j}$ can then be written as

$$
Z_{j}=\operatorname{Ad}(g) X+\operatorname{Ad}\left(g^{\prime}\right) X^{\prime} \bmod \mathfrak{g}_{j+1} .
$$

Let

$$
Y=-\operatorname{Ad}(g) X+\operatorname{Ad}\left(g^{\prime}\right) X^{\prime} .
$$

It is easy to verify then that

$$
\begin{aligned}
\left(Z_{j},-Z_{j}\right) & =(Y, Y)+\left(\operatorname{Ad}(g)(2 X), \operatorname{Ad}\left(g^{\prime}\right)\left(-2 X^{\prime}\right)\right) \quad \bmod \mathfrak{g}_{j+1} \times \mathfrak{g}_{j+1} \\
& \subset \mathfrak{d}+\operatorname{Ad}\left(g, g^{\prime}\right)\left(\mathfrak{g} \times \mathfrak{g}^{\prime}\right) \bmod \mathfrak{g}_{j+1} \times \mathfrak{g}_{j+1} .
\end{aligned}
$$

This is impossible since $\left(g, g^{\prime}\right) \in \mathbb{U}$. Hence $j \in \mathcal{I}\left(\mathfrak{g} / \mathfrak{b}, \mathfrak{g} / \mathfrak{b}^{\prime}\right)$.

Corollary 5.3. Let $\mathcal{U}$ be the nonempty Zariski open subset of $G$ defined in Remark 3.4 for the subgroups $B$ and $B^{\prime}$ and let $\mathbb{U}=\left\{\left(g, g^{\prime}\right) \in \mathbb{G} ; g^{-1} g^{\prime} \in \mathcal{U}\right\}$. Let $\tilde{\mathcal{U}}$ be the projection of $\mathcal{U}$ into $B \backslash G / B^{\prime}$ and $\tilde{\mathbb{U}}$ the projection of $\mathbb{U}$ into $\mathbb{D} \backslash \mathbb{G} / \mathbb{B}$. Then the mapping $\mathbb{M}$ of Proposition 5.1 maps $\tilde{\mathcal{U}}$ homeomorphically onto $\tilde{\mathbb{U}}$.

Now let $\mathcal{U}$ be the Zariski open subset of $G$ with property $(\mathcal{P})$ such that

$$
j \in \mathcal{I}\left(\mathfrak{g} / \mathfrak{b}, \mathfrak{g} / \mathfrak{b}^{\prime}\right) \quad \Longleftrightarrow \quad Z_{j} \notin \mathfrak{b}+\operatorname{Ad}(g) \mathfrak{b}^{\prime}+\mathfrak{g}_{j+1}, \text { for all } g \in \mathcal{U} \text {. }
$$

Let us write

$$
\mathcal{I}\left(\mathfrak{g} / \mathfrak{b}, \mathfrak{g} / \mathfrak{b}^{\prime}\right)=\left\{i_{1}, \cdots, i_{d}\right\} .
$$

Then we have the mapping defined on $\mathbb{R}^{d}$ for the set of double cosets $B \backslash G / B^{\prime}$ as we introduced in Notation 3.5 .

$$
\phi(t)=\exp \left(t_{1} Z_{i_{1}}\right) \cdots \exp \left(t_{d} Z_{i_{d}}\right),
$$

and the equivalent one corresponding to the set of double cosets $\mathbb{D} \backslash \mathbb{G} / \mathbb{B}$ which is $(\phi, \check{\phi})$. In fact,

$$
\exp \left(t_{1}\left(Z_{i_{1}},-Z_{i_{1}}\right)\right) \cdots \exp \left(t_{d}\left(Z_{i_{d}},-Z_{i_{d}}\right)\right)=(\phi(t), \phi(-t)) .
$$

Then the linear functionals $\left(\ell, \ell^{\prime}\right)(t)=\operatorname{Ad}^{*}((\phi, \check{\phi})(t))\left(\ell, \ell^{\prime}\right)_{\mid \mathfrak{d}}$ of $\mathfrak{d}$ that we need as in (4.2), are identified to those on $\mathfrak{g}$ given by

$$
\left(\ell, \ell^{\prime}\right)(t):=\operatorname{Ad}^{*}(\phi(t)) \ell+\operatorname{Ad}^{*}(\phi(-t)) \ell^{\prime} \in \operatorname{Ad}^{*}(G) \ell+\operatorname{Ad}^{*}(G) \ell^{\prime}, t \in \mathbb{R}^{d},
$$

while for $Z \in \mathfrak{g}$, we have

$$
\begin{aligned}
\left\langle\left(\ell, \ell^{\prime}\right)(t),(Z, Z)\right\rangle & =\left\langle\operatorname{Ad}^{*}(\phi(t), \phi(-t))\left(\ell, \ell^{\prime}\right)_{\mid \mathfrak{d}},(Z, Z)\right\rangle \\
& =\left\langle\operatorname{Ad}^{*}(\phi(t)) \ell+\operatorname{Ad}^{*}(\phi(-t)) \ell^{\prime}, Z\right\rangle .
\end{aligned}
$$

We must determine now the index set $I$ defined in (4.5) for the algebras $\mathfrak{b}$ and $\mathfrak{b}^{\prime}$ and the elements $\ell(t, v)$ in $\left(\ell, \ell^{\prime}\right)(t)+\mathcal{B}(t)^{\perp}$ defined in (4.6). 
Remarks 5.4.

(1) Let us remark that the subalgebra $\mathcal{B}(t)$ of $\mathcal{G}$ defined as in Notation 3.13 can be identified to a subalgebra of $\mathfrak{g}$ denoted by $\mathfrak{k}(t)$, while

$$
\mathcal{B}(t)=\mathfrak{d} \cap \operatorname{Ad}(\phi(t))(\mathcal{B}) \simeq \operatorname{Ad}(\phi(t)) b \cap \operatorname{Ad}(\phi(-t)) b^{\prime}=: \mathfrak{k}(t) .
$$

(2) Since $\operatorname{Ad}(\phi(t)) \mathfrak{b}$ is a polarization at $\operatorname{Ad}^{*}(\phi(t)) \ell$ and similarly for $\operatorname{Ad}(\phi(-t)) \mathfrak{b}^{\prime}$, we have that

$$
\begin{aligned}
\left(\ell, \ell^{\prime}\right)(t)+\mathfrak{k}(t)^{\perp} & =\operatorname{Ad}^{*}(\phi(t)) \ell+\operatorname{Ad}^{*}(\phi(-t)) \ell^{\prime}+\left(\operatorname{Ad}(\phi(t)) \mathfrak{b} \cap \operatorname{Ad}(\phi(-t)) \mathfrak{b}^{\prime}\right)^{\perp} \\
& =\operatorname{Ad}^{*}(\phi(t)) \ell+\operatorname{Ad}^{*}(\phi(-t)) \ell^{\prime}+\operatorname{Ad}(\phi(t)) \mathfrak{b}^{\perp}+\operatorname{Ad}(\phi(-t)) \mathfrak{b}^{\perp} \\
& =\operatorname{Ad}^{*}\left(\phi(t) B \phi(t)^{-1}\right) \ell+\operatorname{Ad}^{*}\left(\phi(-t) B^{\prime} \phi(-t)^{-1}\right) \ell^{\prime} \\
& \subset \operatorname{Ad}^{*}(G) \ell+\operatorname{Ad}^{*}(G) \ell^{\prime} .
\end{aligned}
$$

Hence, the affine subsets $\mathcal{R}(t)$ defined in (4.4) are all contained in the sum of the orbits $\Omega_{\pi}+\Omega_{\pi^{\prime}}$ of $\ell$ and of $\ell^{\prime}$.

Notation 5.5. Let us also denote for $t \in \mathcal{V}$,

$$
\mathfrak{p}(t):=\operatorname{Ad}(\phi(t)) \mathfrak{b}, \quad \mathfrak{p}^{\prime}(t):=\operatorname{Ad}(\phi(-t)) \mathfrak{b}^{\prime} .
$$

Then $\mathfrak{p}(t)$ is a polarization at $\operatorname{Ad}^{*}(\phi(t)) \ell$ and $\mathfrak{p}^{\prime}(t)$ at $\operatorname{Ad}^{*}(\phi(-t)) \ell^{\prime}$. When we put $P(t)=\exp \mathfrak{p}(t)$ and $P^{\prime}(t)=\exp \mathfrak{p}^{\prime}(t)$ for $t \in \mathcal{V}$, then the subalgebra of their intersection $P(t) \cap P^{\prime}(t)$ is not in general a polarization of $\mathfrak{g}$ at $\left(\ell, \ell^{\prime}\right)(t)$. That's why we have to disintegrate the unitary representation

$$
\sigma(t)=\operatorname{ind}_{P(t) \cap P^{\prime}(t)}^{G} \chi_{\left(\ell, \ell^{\prime}\right)(t)}:=\sigma(x) \quad\left(\text { where } x=\tilde{\phi}(t) \in B \backslash G / B^{\prime}\right)
$$

into irreducibles of $G$. In fact, we view it as the unitary representation of $\mathbb{G}$ given by

$$
\begin{aligned}
\sigma(\tilde{x}):=\operatorname{ind}_{P(t) \times P^{\prime}(t)}^{G \times G} \chi_{\left(\ell, \ell^{\prime}\right)(t)} \\
\\
\left(\text { where } \tilde{x}=\mathbb{M}(x) \in \mathbb{D} \backslash \mathbb{G} / \mathbb{B} \text { for } x=\tilde{\phi}(t) \in B \backslash G / B^{\prime}\right) .
\end{aligned}
$$

From Proposition 3.27, we deduce that

$$
\left(\pi \times \pi^{\prime}\right)_{\mid \mathbb{D}} \simeq \int_{\mathbb{D} \backslash \mathbb{G} / \mathbb{B}}^{\oplus} \sigma(\tilde{x}) d \gamma(\tilde{x}) .
$$

Thus, we obtain the first writing of the disintegration of $\pi \otimes \pi^{\prime}$ :

$$
\begin{aligned}
\pi \otimes \pi^{\prime} & \simeq \int_{B \backslash G / B^{\prime}}^{\oplus} \sigma(x) d \gamma(x) \\
& \simeq \int_{\mathcal{V}}^{\oplus} \sigma(t)|F(t)| d t
\end{aligned}
$$

where $F$ is the function defined as in Definition 3.23 (for the subgroups $B$ and $B^{\prime}$ ).

To obtain the final decomposition, we have just to repeat the process developed in the fourth section. So, we consider the Zariski open subset $\mathcal{W} \subset \mathbb{R}^{d} \times \mathbb{R}^{q}$ 
contained in $\bigcup_{t \in \mathcal{V}}^{\cdot} \mathcal{R}(t)$ and we put for $w=(t, v) \in \mathcal{W}$ with $t \in \mathcal{V}, v \in \mathbb{R}^{q}$ and $x=\tilde{\phi}(t) \in B \backslash G / B^{\prime}$,

- the linear functional $f_{w}=f_{(x, v)} \in \mathcal{R}(x)=\mathcal{R}(t)$ as in (4.6),

$$
\begin{aligned}
f_{w} & =\ell(t, v)=\ell(t)+\sum_{c \in I(t)} v_{i} Y_{j_{c}}(t)^{*} \\
& \in \Omega_{\pi}+\Omega_{\pi^{\prime}} \quad(\text { by Remark [5.4.2), }
\end{aligned}
$$

- the unitary irreducible representation of $G$ as in (4.7),

$$
\rho_{w}=\operatorname{ind}_{K(w)}^{G} \chi_{f_{w}}=: \rho_{(x, v)}
$$

where $\mathfrak{k}(w)=\operatorname{Lie}(K(w))$ is here the Vergne polarization of $\mathfrak{g}$ at $f_{w}$,

- and we define the measure $d \omega$ on $\Omega_{\pi}+\Omega_{\pi^{\prime}}$ as the image of $d w=|F(t)| d t \otimes d v$ on $\mathbb{R}^{d} \times \mathbb{R}^{q}$ through the mapping $\mathcal{W} \ni w \mapsto \omega:=f_{w} \in \Omega_{\pi}+\Omega_{\pi^{\prime}}$.

We can now apply Theorem 4.2 for the special case of $\Pi$ and $\mathbb{D}$. We obtain an operator

$$
\mathcal{T}: L^{2}\left(\mathbb{G} / \mathbb{B}, \chi_{\left(\ell, \ell^{\prime}\right)}\right) \longrightarrow \int_{\mathcal{W}}^{\oplus} L^{2}\left(G / K(w), \chi_{f_{w}}\right) d w
$$

between the tensor product and its disintegration into irreducibles of $G$,

$$
\pi \otimes \pi^{\prime} \simeq \int_{B \backslash G / B^{\prime}}^{\oplus}\left(\int_{\mathcal{R}(x)}^{\oplus} \rho_{(x, v)} d v\right) d \gamma(x)
$$

By considering the previous computations, this decomposition can be expressed differently; in fact

$$
\pi \otimes \pi^{\prime} \simeq \int_{\Omega_{\pi}+\Omega_{\pi^{\prime}}}^{\oplus} \rho(\omega) d \omega \simeq \int_{\mathcal{W}}^{\oplus} \rho_{w} d w
$$

and the operator $\mathcal{T}$ is at first defined on $\mathcal{S}\left(\mathbb{G} / \mathbb{B}, \chi_{\left(\ell, \ell^{\prime}\right)}\right)$ as follows. For every $\xi \in \mathcal{S}\left(\mathbb{G} / \mathbb{B}, \chi_{\left(\ell, \ell^{\prime}\right)}\right)$, we put $\mathcal{T}(\xi): \mathcal{W} \ni w \longmapsto \mathcal{T} \xi(w) \in L^{2}\left(G / K(w), \chi_{f_{w}}\right)$, such that for any $w=(t, v) \in \mathcal{W}$,

$$
\mathcal{T} \xi(w): G \ni g \longmapsto \int_{K(w) /\left(K(w) \cap P(t) \cap P^{\prime}(t)\right)} \xi(g b \phi(t), g b \phi(-t)) \chi_{f_{w}}(b) d_{\mathcal{Y}(w)}(b) \in \mathbb{C}
$$

where $\mathcal{Y}(w)$ is as in (4.8). Then $\mathcal{T}$ extends to a unitary intertwining operator and one has

$$
\int_{\mathcal{W}}\|\mathcal{T} \xi(w)\|_{L^{2}\left(G / K(w), \chi_{f_{w}}\right)}^{2} d w=\|\xi\|_{L^{2}\left(\mathbb{G} / \mathbb{B}, \chi_{\left(\ell, \ell^{\prime}\right)}\right)}^{2} .
$$

Theorem 5.6. Let $G=\exp \mathfrak{g}$ be a connected simply connected nilpotent Lie group and $\mathcal{Z}$ a Jordan-Hölder basis of $\mathfrak{g}$. Let $\ell \in \mathfrak{g}^{*}$ (respectively $\ell^{\prime} \in \mathfrak{g}^{*}$ ) and $\mathfrak{b}$ (respectively $\left.\mathfrak{b}^{\prime}\right)$ be the Vergne polarization at $\ell$ (respectively at $\left.\ell^{\prime}\right)$, with respect to $\mathcal{Z}$. We realize $\pi \in \hat{G}$ as $\pi_{\ell, \mathfrak{b}}=\operatorname{ind}_{B}^{G} \chi_{\ell}$ where $B=\exp (\mathfrak{b})$, and similarly for $\pi^{\prime} \in \hat{G}$. Then their tensor product is a direct integral over the sum of orbits $\Omega_{\pi}+\Omega_{\pi^{\prime}}$ (with respect to the measure $d \omega)$. Finally, an explicit unitary intertwining operator $\mathcal{T}$ which decomposes $\pi \otimes \pi^{\prime}$ as in Reference (5.6) is provided by the formula (5.7). 


\section{An EXAMPLE}

We show in the following example that the choice of a polarization for a given linear functional has a strong influence on the concrete form of the disintegration of the representation $\pi \otimes \pi^{\prime}$.

Let $\mathfrak{g}$ be the Lie algebra spanned by the vectors $\left\langle Z_{1}, \cdots, Z_{7}\right\rangle$ and equipped with the nontrivial brackets

$$
\left[Z_{1}, Z_{4}\right]=Z_{7}, \quad\left[Z_{3}, Z_{4}\right]=Z_{6}, \quad\left[Z_{2}, Z_{4}\right]=Z_{5}, \quad\left[Z_{3}, Z_{5}\right]=Z_{7} . \quad\left[Z_{2}, Z_{6}\right]=Z_{7},
$$

Then $\mathcal{Z}=\left\{Z_{1}, \cdots, Z_{7}\right\}$ is a Jordan-Hölder basis of $\mathfrak{g}$ and one can easily verify that the coadjoint orbit $G \cdot \ell$ of any $\ell \in \mathfrak{g}^{*}$ with $\ell\left(Z_{7}\right) \neq 0$ is equal to $\ell+Z_{7}^{\perp}$. Let $\mathcal{Z}^{*}=\left\{Z_{1}^{*}, \cdots, Z_{7}^{*}\right\}$ be the dual basis of $\mathcal{Z}$.

Now choose $\ell=Z_{7}^{*}+Z_{6}^{*} \in \mathfrak{g}^{*}$. Its stabilizer in $\mathfrak{g}$ is reduced to the center $\mathbb{R} Z_{7}$ of $\mathfrak{g}$. There are several possibilities for polarizations at $\ell$. We take 4 such polarizations:

$$
\begin{aligned}
& \mathfrak{b}_{1}:=\operatorname{span}\left\langle Z_{7}, Z_{6}, Z_{5}, Z_{4}\right\rangle, \\
& \mathfrak{b}_{2}:=\operatorname{span}\left\langle Z_{7}, Z_{3}, Z_{2}, Z_{1}\right\rangle, \\
& \mathfrak{b}_{3}:=\operatorname{span}\left\langle Z_{7}, Z_{6}, Z_{4}-Z_{5}, Z_{3}\right\rangle, \\
& \mathfrak{b}_{4}:=\operatorname{span}\left\langle Z_{7}, Z_{6}, Z_{4}, Z_{1}\right\rangle .
\end{aligned}
$$

Then $\mathfrak{b}_{1}$ is the Vergne polarization associated to the Jordan-Hölder basis $\mathcal{Z}$. It is also an abelian ideal of $\mathfrak{g}$. $\mathfrak{b}_{2}$ and $\mathfrak{b}_{4}$ are also abelian subalgebras but not $\mathfrak{b}_{3}$.

Now let

$$
\pi_{j}:=\operatorname{ind}_{B_{j}}^{G} \chi_{\ell}, j=1,2,3,4 .
$$

6.1. Disintegration of $\pi_{1} \otimes \pi_{2}$. We have that $\mathfrak{b}_{1}$ is an ideal of $\mathfrak{g}$ and $\mathfrak{b}_{1}+\mathfrak{b}_{2}=\mathfrak{g}$. Hence $\mathcal{I}\left(\mathfrak{g} / \mathfrak{b}_{1}, \mathfrak{g} / \mathfrak{b}_{2}\right)=\emptyset$. Hence by (5.5)), we have that

$$
\pi_{1} \otimes \pi_{2} \simeq \sigma(0)=\operatorname{ind}_{B_{1} \cap B_{2}}^{G} \chi_{2 \ell} .
$$

We must determine the index set $I$ of (4.3). We have now that $2 \ell+\left(\mathfrak{b}_{1} \cap \mathfrak{b}_{2}\right)^{\perp}=$ $2 \ell+Z_{7}^{\perp}$ and the family $\left\{Z_{1}, \cdots, Z_{6}\right\}$ is a Malcev basis of $\mathfrak{g}$ relative to $\mathfrak{b}_{1} \cap \mathfrak{b}_{2}$. We must determine the indices set $I$. We have for a linear form $f=2 Z_{7}^{*}+\sum_{j=2}^{1} \beta Z_{j}^{*} \in$ $2 \ell+\mathcal{Z}_{7}^{\perp}$ that

$$
\begin{array}{rlrl}
\mathfrak{g}_{6}\left(f_{6}\right) & =\mathfrak{g}_{6} & \nsubseteq \mathfrak{g}_{7} & \Longrightarrow 6 \in I, \\
\mathfrak{g}_{5}\left(f_{5}\right) & =\mathfrak{g}_{5} & \nsubseteq \mathfrak{g}_{6} \Longrightarrow 5 \in I, \\
\mathfrak{g}_{4}\left(f_{4}\right) & =\mathfrak{g}_{4} & \Longrightarrow & \Longrightarrow \in \in, \\
\mathfrak{g}_{3}\left(f_{3}\right) & =\operatorname{span}\left\langle Z_{7}, Z_{6}, Z_{4}-\frac{\beta_{6}}{2} Z_{5}\right\rangle & \subset \mathfrak{g}_{4} & \Longrightarrow 3 \notin I, \\
\mathfrak{g}_{2}\left(f_{2}\right) & =\operatorname{span}\left\langle Z_{7}, Z_{4}-\frac{\beta_{6}}{2} Z_{5}+\frac{\beta_{6}}{2} Z_{6}\right\rangle & \subset \mathfrak{g}_{3} & \Longrightarrow 2 \notin I, \\
\mathfrak{g}(f) & =\mathfrak{g}_{7} & \subset \mathfrak{g}_{2} & \Longrightarrow 1 \notin I,
\end{array}
$$

where $f_{j}:=f_{\mid \mathfrak{g}_{j}}$. This implies that $I=\left\{k_{1}=4, k_{2}=5, k_{3}=6\right\}$ and the space $\mathcal{R}$ of disintegration of $\pi_{1} \otimes \pi_{2}$ is the space of $\sigma(0)$ given by $\mathcal{R}(0)=2 Z_{7}^{*}+\mathbb{R} Z_{6}^{*}+$ $\mathbb{R} Z_{5}^{*}+\mathbb{R} Z_{4}^{*}$. We can take for every $w=\left(v_{4}, v_{5}, v_{6}\right) \in \mathcal{W}=\mathbb{R}^{3}$ the Vergne polarization $\mathfrak{b}=\mathfrak{b}_{1}$ associated to the Jordan-Hölder basis $\mathcal{Z}$ at $f_{w} \in \mathcal{R}$. The mapping $T_{w}: L^{2}\left(\mathbb{G} / B_{1} \times B_{2}, \chi_{\ell, \ell}\right)$ of Theorem 5.6 is then given for $g \in G$ by

$$
\begin{aligned}
& T_{w}(\xi)(g) \\
& =\int_{\mathbb{R}^{3}} \xi\left(g\left(u_{4} Z_{4} \cdot u_{5} Z_{5} \cdot u_{6} Z_{6}\right), g\left(u_{4} Z_{4} \cdot u_{5} Z_{5} \cdot u_{6} Z_{6}\right)\right) e^{-2 \pi i\left(v_{4} u_{4}+v_{5} u_{5}+\left(v_{6}+1\right) u_{6}+2 Z_{7}^{*}\right)} d u,
\end{aligned}
$$


and

$$
\mathcal{H}_{\pi_{1}} \hat{\otimes} \mathcal{H}_{\pi_{2}} \simeq \int_{\mathbb{R}^{3}}^{\oplus} L^{2}\left(G / B, \chi_{v_{4} Z_{4}^{*}+v_{5} Z_{5}^{*}+\left(v_{6}+1\right) Z_{6}^{*}+2 Z_{7}^{*}}\right) d u_{4} d u_{5} d u_{6}
$$

6.2. Disintegration of $\pi_{3} \otimes \pi_{4}$. We have here that

$$
\mathcal{I}^{\mathfrak{g} / \mathfrak{b}_{3}}=\{1,2,5\}, \quad \mathcal{I}^{\mathfrak{g} / \mathfrak{b}_{4}}=\{2,3,5\}
$$

and so $I^{\mathfrak{g} / \mathfrak{b}_{3}} \cap \mathcal{I}^{\mathfrak{g} / \mathfrak{b}_{4}}=\{2,5\}$.

Since for every $g=\prod_{j=1}^{7}\left(g_{j} Z_{j}\right), g_{2} \neq 0$,

$$
\begin{aligned}
Z_{5}=Z_{5}-Z_{4}+\operatorname{Ad}\left(\left(g_{1} Z_{1}\right)\left(g_{2} Z_{2}\right)\left(g_{3} Z_{3}\right)\left(g_{4} Z_{4}\right)\right)\left(\frac{1}{g_{2}}\left(-Z_{4}\right)\right) \\
\quad \bmod \mathfrak{g}_{6} \subset \mathfrak{b}_{3}+\operatorname{Ad}(g)\left(\mathfrak{b}_{4}\right) \bmod \mathfrak{g}_{6},
\end{aligned}
$$

it follows that $5 \notin \mathcal{I}\left(\mathfrak{g} / \mathfrak{b}_{3}, \mathfrak{g} / \mathfrak{b}_{4}\right)$. Hence, since $[\mathfrak{g}, \mathfrak{g}]=\operatorname{span}\left\langle Z_{5}, Z_{6}, Z_{7}\right\rangle$, we have that

$$
\mathcal{I}\left(\mathfrak{g} / \mathfrak{b}_{3}, \mathfrak{g} / \mathfrak{b}_{4}\right)=\{2\} .
$$

We must compute the function $F$ of Reference (3.23). Let $\Phi: \mathbb{R}^{2} \times \mathbb{R} \longrightarrow G / B_{4}$ be the mapping such that

$$
\Phi\left(s_{1}, s_{2}, t\right)=\left(s_{1} Z_{1} \cdot s_{2}\left(Z_{4}-Z_{5}\right) \cdot t Z_{2}\right) \cdot B_{4}=\left(s_{1} Z_{1} \cdot t Z_{2} \cdot\left(s_{2} t+1\right) Z_{5}\right) \cdot B_{4} .
$$

Therefore

$$
\Phi\left(s_{1}, s_{2}, t\right) \equiv\left(s_{1}, t, 1+s_{2} t\right)
$$

and so

$$
F(t)=t .
$$

We deduce from (5.5) the first step of the disintegration

$$
\pi_{3} \otimes \pi_{4} \simeq \int_{\mathbb{R}}^{\oplus} \sigma(t)|t| d t
$$

where $\sigma(t)=\sigma\left(B_{3} \cdot \exp \left(t Z_{2}\right) \cdot B_{4}\right)=\operatorname{ind}_{P(t) \cap P^{\prime}(t)}^{G} \chi\left(\ell, \ell^{\prime}\right)(t)$ has $\mathcal{R}(t)=2 Z_{7}^{*}+\mathbb{R} Z_{5}^{*}+$ $\mathbb{R} Z_{4}^{*}$ as the space of disintegration for every $t \in \mathbb{R}$. In fact, we have that

- $\operatorname{Ad}^{*}\left(t Z_{2}\right) \ell=\ell-t Z_{6}^{*} \Rightarrow \ell(t)=\operatorname{Ad}^{*}\left(t Z_{2}\right) \ell+\operatorname{Ad}^{*}\left(-t Z_{2}\right) \ell=2 \ell, t \in \mathbb{R}$,

- the Lie algebra $\left(\mathfrak{p}(t) \cap \mathfrak{p}^{\prime}(t)\right)$ of Reference (5.3) is given for $t \in \mathbb{R}$ by

$$
\operatorname{Ad}\left(t Z_{2}\right) \mathfrak{b}_{3} \cap \operatorname{Ad}\left(-t Z_{2}\right) \mathfrak{b}_{4}=\operatorname{span}\left\{Z_{6}, Z_{7}\right\} .
$$

Hence the space of disintegration is this time $\mathcal{W}=\mathbb{R} \times \mathbb{R}^{2}$ and for $w=\left(t,\left(v_{4}, v_{5}\right)\right) \in$ $\mathcal{W}$, the mapping $T_{w}$ is defined by

$$
T_{w} \xi(g)=\int_{\mathbb{R}^{2}} \xi\left(g\left(u_{4} Z_{4} \cdot u_{5} Z_{5} \cdot t Z_{2}\right), g\left(u_{4} Z_{4} \cdot u_{5} Z_{5} \cdot\left(-t Z_{2}\right)\right) e^{-2 \pi i\left(u_{4} v_{4}+u_{5} v_{5}\right)} d u_{4} d u_{5}\right.
$$

and

$$
\mathcal{H}_{\pi_{3}} \hat{\otimes} \mathcal{H}_{\pi_{4}} \simeq \int_{\mathbb{R}}^{\oplus} \int_{\mathbb{R}^{2}}^{\oplus} L^{2}\left(G / B, \chi_{v_{4} Z_{4}^{*}+v_{5} Z_{5}^{*}+2 Z_{7}^{*}}\right)|t| d v_{4} d v_{5} d t
$$




\section{CRIteria FOR IRREDUCIBILITY}

In this section we shall determine a necessary and sufficient condition for the irreducibility of the tensor representation $\pi \otimes \pi^{\prime}$. We shall prove the following theorem.

Theorem 7.1. Let $G=\exp \mathfrak{g}$ be a simply connected nilpotent Lie group, $\pi=\pi_{\ell}$ and $\pi^{\prime}=\pi_{\ell^{\prime}}$ two irreducible unitary representations of $G$. The following assertions are equivalent.

(1) $\pi \otimes \pi^{\prime} \in \hat{G}$.

(2) For any polarizations $\mathfrak{b}$ at $\ell$ and $\mathfrak{b}^{\prime}$ at $\ell^{\prime}$, we have that

$$
\left\{\begin{array}{c}
\mathfrak{b}+\mathfrak{b}^{\prime}=\mathfrak{g} \text { and } \\
\mathfrak{b} \cap \mathfrak{b}^{\prime} \text { is a polarization at } \ell+\ell^{\prime} .
\end{array}\right.
$$

(3) There exist some polarizations $\mathfrak{b}$ at $\ell$ and $\mathfrak{b}^{\prime}$ at $\ell^{\prime}$ such that

$$
\left\{\begin{array}{c}
\mathfrak{b}+\mathfrak{b}^{\prime}=\mathfrak{g} \text { and } \\
\mathfrak{b} \cap \mathfrak{b}^{\prime} \text { is a polarization at } \ell+\ell^{\prime},
\end{array}\right.
$$

$$
\operatorname{Ad}^{*}(G) \ell+\operatorname{Ad}^{*}(G) \ell^{\prime}=\operatorname{Ad}^{*}(G)\left(\ell+\ell^{\prime}\right)
$$

and

$$
\mathfrak{g}(\ell)+\mathfrak{g}\left(\ell^{\prime}\right)=\mathfrak{g}
$$

Proof. 1) $\Longrightarrow 2$ )

By (5.4), the space of double cosets $\mathbb{D} \backslash \mathbb{G} / \mathbb{B}$ must be reduced to one point. This is then the case of $B \backslash G / B^{\prime}$ while they are in bijection as we mentioned in Proposition 5.1. This implies by Lemma 3.9 that $\mathcal{I}\left(\mathfrak{g} / \mathfrak{b}, \mathfrak{g} / \mathfrak{b}^{\prime}\right)=\emptyset$ and $G=$ $B B^{\prime}$. In particular this tells us that $L^{2}\left(\mathbb{G} / \mathbb{B}, \chi_{\left(\ell, \ell^{\prime}\right)}\right)$ is isometrically isomorphic to $L^{2}\left(G / B \cap B^{\prime}, \chi_{\ell+\ell^{\prime}}\right)$. In fact, we have constructed in the proof of Proposition 3.27 the isometric operator $S$ which, in our case here, maps $L^{2}\left(\mathbb{G} / \mathbb{B}, \chi_{\left(\ell, \ell^{\prime}\right)}\right)$ to $L^{2}\left(\Delta G / B \times B^{\prime}, \chi_{\left(\ell, \ell^{\prime}\right)}\right)$, but from Remarks 5.4(1), we see that this last space is isometrically isomorphic to $L^{2}\left(G / B \cap B^{\prime}, \chi_{\ell+\ell^{\prime}}\right)$, and so $\pi \otimes \pi^{\prime} \simeq \operatorname{ind}_{B \cap B^{\prime}}^{G} \chi \ell+\ell^{\prime}$. Hence the representation $\operatorname{ind}_{B \cap B^{\prime}}^{G} \chi_{\ell+\ell^{\prime}}$ is irreducible, which forces $\mathfrak{b} \cap \mathfrak{b}^{\prime}$ to be a polarization at $\ell+\ell^{\prime}$ by Kirillov's theorem.

2) $\Longrightarrow 3$ ) is trivial.

3) $\Longrightarrow 4$ )

Let us first show that $\operatorname{Ad}^{*}(G) \ell+\operatorname{Ad}^{*}(G) \ell^{\prime}=\operatorname{Ad}^{*}(G)\left(\ell+\ell^{\prime}\right)$. We do this by an induction on $\operatorname{dim}(\mathfrak{g})$. If this dimension is 1 , the implication is evident, since we work then with characters. If $\ell^{\prime}$ is a character, then we always have that $\operatorname{Ad}^{*}(G) \ell+$ $\operatorname{Ad}^{*}(G) \ell^{\prime}=\operatorname{Ad}^{*}(G) \ell+\ell^{\prime}=\operatorname{Ad}^{*}(G)\left(\ell+\ell^{\prime}\right)$. We can thus assume that $\mathfrak{b}^{\prime}$ is a proper subalgebra of $\mathfrak{g}$. Let $\mathfrak{h}$ be again an ideal of codimension 1 in $\mathfrak{g}$ containing $\mathfrak{b}^{\prime}$. Then $\mathfrak{b} \not \subset \mathfrak{h}$ by the condition $\mathfrak{b}+\mathfrak{b}^{\prime}=\mathfrak{g}$. Hence $\mathfrak{b} \cap \mathfrak{h}$ is a polarization at $\ell_{\mid \mathfrak{h}}$ and $\mathfrak{b}^{\prime}$ is a polarization at $\ell_{\mid \mathfrak{h}}^{\prime}$. Furthermore, we can also see easily that their sum $\mathfrak{b} \cap \mathfrak{h}+\mathfrak{b}^{\prime}$ is $\mathfrak{h}$ entire, and that their intersection $(\mathfrak{b} \cap \mathfrak{h}) \cap \mathfrak{b}^{\prime}=\mathfrak{b} \cap \mathfrak{b}^{\prime}$ is a polarization of $\mathfrak{h}$ at $\ell_{\mid h}+\ell_{\mid \mathfrak{h}}^{\prime}$, but also a polarization of $\mathfrak{g}$ at $\ell+\ell^{\prime}$ too, which means that the $G$-orbit $\operatorname{Ad}^{*}(G)\left(\ell+\ell^{\prime}\right)$ is saturated with respect to $\mathfrak{h}$. It follows from the induction hypothesis that

$$
\operatorname{Ad}^{*}(H) \ell_{\mid \mathfrak{h}}+\operatorname{Ad}^{*}(H) \ell_{\mid \mathfrak{h}}^{\prime}=\operatorname{Ad}^{*}(H)\left(\ell_{\mid \mathfrak{h}}+\ell_{\mid \mathfrak{h}}^{\prime}\right)
$$


Therefore

$$
\operatorname{Ad}^{*}(H) \ell+\operatorname{Ad}^{*}(H) \ell^{\prime}+\mathfrak{h}^{\perp}=\operatorname{Ad}^{*}(H)\left(\ell+\ell^{\prime}\right)+\mathfrak{h}^{\perp} .
$$

Now, since the $G$-orbit $\operatorname{Ad}^{*}(G)(\ell)$ is nonsaturated with respect to $\mathfrak{h}$ contrary to $\operatorname{Ad}^{*}(G)\left(\ell^{\prime}\right)$ and $\operatorname{Ad}^{*}(G)\left(\ell+\ell^{\prime}\right)$, they replace respectively $\operatorname{Ad}^{*}(H) \ell, \operatorname{Ad}^{*}(H) \ell^{\prime}+\mathfrak{h}^{\perp}$ and $\operatorname{Ad}^{*}(H)\left(\ell+\ell^{\prime}\right)+\mathfrak{h}^{\perp}$. Finally we obtain

$$
\operatorname{Ad}^{*}(G) \ell+\operatorname{Ad}^{*}(G) \ell^{\prime}=\operatorname{Ad}^{*}(G)\left(\ell+\ell^{\prime}\right) .
$$

Let us show now that $\mathfrak{g}(\ell)+\mathfrak{g}\left(\ell^{\prime}\right)=\mathfrak{g}$.

We have for any polarization $\mathfrak{b}$ at $\ell$ that

$$
\operatorname{dim}(\mathfrak{b})=\frac{\operatorname{dim}(\mathfrak{g})+\operatorname{dim}(\mathfrak{g}(\ell))}{2} .
$$

Hence, since $\mathfrak{b}+\mathfrak{b}^{\prime}=\mathfrak{g}$ and $\mathfrak{b} \cap \mathfrak{b}^{\prime}$ is a polarization at $\ell+\ell^{\prime}$ and since

$$
\operatorname{dim}(\mathfrak{b})+\operatorname{dim}\left(\mathfrak{b}^{\prime}\right)=\operatorname{dim}(\mathfrak{g})+\operatorname{dim}\left(\mathfrak{b} \cap \mathfrak{b}^{\prime}\right),
$$

we have that

$$
\frac{2 \operatorname{dim}(\mathfrak{g})+\operatorname{dim}(\mathfrak{g}(\ell))+\operatorname{dim}\left(\mathfrak{g}\left(\ell^{\prime}\right)\right)}{2}=\operatorname{dim}(\mathfrak{g})+\frac{\operatorname{dim}(\mathfrak{g})+\operatorname{dim}\left(\mathfrak{g}\left(\ell+\ell^{\prime}\right)\right)}{2} .
$$

Therefore, since $\mathfrak{g}(\ell) \cap \mathfrak{g}\left(\ell^{\prime}\right) \subset \mathfrak{g}\left(\ell+\ell^{\prime}\right)$,

$$
\begin{aligned}
\operatorname{dim}\left(\mathfrak{g}(\ell)+g\left(\ell^{\prime}\right)\right)+\operatorname{dim}\left(\mathfrak{g}\left(\ell+\ell^{\prime}\right)\right) & \geq \operatorname{dim}\left(\mathfrak{g}(\ell)+g\left(\ell^{\prime}\right)\right)+\operatorname{dim}\left(\mathfrak{g}(\ell) \cap \mathfrak{g}\left(\ell^{\prime}\right)\right) \\
& =\operatorname{dim}(\mathfrak{g}(\ell))+\operatorname{dim}\left(\mathfrak{g}\left(\ell^{\prime}\right)\right) \\
& =\operatorname{dim}\left(\mathfrak{g}\left(\ell+\ell^{\prime}\right)\right)+\operatorname{dim}(\mathfrak{g}),
\end{aligned}
$$

and so $\operatorname{dim}\left(\mathfrak{g}(\ell)+\mathfrak{g}\left(\ell^{\prime}\right)\right) \geq \operatorname{dim}(\mathfrak{g})$, which implies that $\mathfrak{g}=\mathfrak{g}(\ell)+\mathfrak{g}\left(\ell^{\prime}\right)$.

4) $\Longrightarrow 1)$

Let $\mathfrak{b}\left(\right.$ resp. $\left.\mathfrak{b}^{\prime}\right)$ be a polarization at $\ell$ (resp. at $\left.\ell^{\prime}\right)$. Then $\mathfrak{g}(\ell) \subset \mathfrak{b}, \mathfrak{g}\left(\ell^{\prime}\right) \subset \mathfrak{b}^{\prime}$ and hence $\mathfrak{b}+\mathfrak{b}^{\prime}=\mathfrak{g}$. Therefore $B B^{\prime}$ contains an open subset of $G$, whence it is itself open. Since the product of two closed connected subgroups of $G$ is always closed, it follows that $B B^{\prime}$ is open and closed, which implies that $B B^{\prime}=G$. Therefore $\pi_{\ell, \mathfrak{b}} \otimes$ $\pi_{\ell^{\prime}, \mathfrak{b}^{\prime}} \simeq \operatorname{ind}_{B \cap B^{\prime}}^{G} \chi_{\ell+\ell^{\prime}}$. If we can show that $\mathfrak{b} \cap \mathfrak{b}^{\prime}$ is a polarization at $\ell+\ell^{\prime}$, then we know that $\pi \otimes \pi^{\prime}$ is irreducible. The condition $\operatorname{Ad}^{*}(G) l+\operatorname{Ad}^{*}(G) l^{\prime}=\operatorname{Ad}^{*}(G)\left(l+l^{\prime}\right)$ implies that the sum of the tangent spaces $\operatorname{ad}^{*}(\mathfrak{g}) l$ and $\operatorname{ad}^{*}(\mathfrak{g}) l^{\prime}$ of the two orbits $\operatorname{Ad}^{*}(G) l$ and $\operatorname{Ad}^{*}(G) l^{\prime}$ is contained in the tangent space $\operatorname{ad}^{*}(\mathfrak{g})\left(l+l^{\prime}\right)$ of the orbit $\operatorname{Ad}^{*}(G)\left(l+l^{\prime}\right)$. Furthermore if for some $X \in \mathfrak{g}(l)$ and some $X^{\prime} \in \mathfrak{g}\left(l^{\prime}\right)$ we have that $\operatorname{ad}^{*}(X) l^{\prime}=\operatorname{ad}^{*}\left(-X^{\prime}\right) l=0$, then $\operatorname{ad}^{*}\left(X^{\prime}\right) l \in \mathfrak{g}(l)^{\perp} \cap \mathfrak{g}\left(l^{\prime}\right)^{\perp}=\left(\mathfrak{g}(l)+\mathfrak{g}\left(l^{\prime}\right)\right)^{\perp}=\mathfrak{g}^{\perp}=$ $\{0\}$. Hence the mapping

$\operatorname{ad}^{*}(\mathfrak{g}) l^{\prime} \times \operatorname{ad}^{*}(\mathfrak{g}) l \rightarrow \operatorname{ad}^{*}(\mathfrak{g})\left(l+l^{\prime}\right),\left(\operatorname{ad}^{*}\left(X^{\prime}\right) l, \operatorname{ad}^{*}(X) l^{\prime}\right) \mapsto \operatorname{ad}^{*}\left(X^{\prime}\right) l+\operatorname{ad}^{*}(X) l^{\prime}$

is injective. Therefore

$$
\operatorname{dim}(\mathfrak{g} / \mathfrak{g}(l))+\operatorname{dim}\left(\mathfrak{g} / \mathfrak{g}\left(l^{\prime}\right)\right) \leq \operatorname{dim}\left(\mathfrak{g} / \mathfrak{g}\left(l+l^{\prime}\right)\right) .
$$

Hence

$$
\begin{aligned}
2 \operatorname{dim}(\mathfrak{g})-\operatorname{dim}(\mathfrak{g}(l))-\operatorname{dim}(\mathfrak{g}(l)) & \leq \operatorname{dim}(\mathfrak{g})-\operatorname{dim}\left(\mathfrak{g}\left(l+l^{\prime}\right)\right) \\
& \Rightarrow \\
\operatorname{dim}\left(\mathfrak{g}(l)+\mathfrak{g}\left(l^{\prime}\right)\right)-\operatorname{dim}(\mathfrak{g}(l))-\operatorname{dim}(\mathfrak{g}(l)) & \leq-\operatorname{dim}(\mathfrak{g})-\operatorname{dim}\left(\mathfrak{g}\left(l+l^{\prime}\right)\right) \\
& \Rightarrow \\
\operatorname{dim}\left(\mathfrak{g}\left(l+l^{\prime}\right)\right) & \leq \operatorname{dim}\left(\mathfrak{g}(l) \cap \mathfrak{g}\left(l^{\prime}\right)\right) .
\end{aligned}
$$


Therefore $\mathfrak{g}(l) \cap \mathfrak{g}\left(l^{\prime}\right)=\mathfrak{g}\left(l+l^{\prime}\right)$, since $\mathfrak{g}(l) \cap \mathfrak{g}\left(l^{\prime}\right) \subset \mathfrak{g}\left(l+l^{\prime}\right)$.

This tells us then that

$$
\begin{aligned}
\operatorname{dim}\left(\mathfrak{b} \cap \mathfrak{b}^{\prime}\right) & =\operatorname{dim}(\mathfrak{b})+\operatorname{dim}\left(\mathfrak{b}^{\prime}\right)-\operatorname{dim}\left(\mathfrak{b}+\mathfrak{b}^{\prime}\right) \\
& =\operatorname{dim}(\mathfrak{b})+\operatorname{dim}\left(\mathfrak{b}^{\prime}\right)-\operatorname{dim}(\mathfrak{g}) \\
& =\frac{2 \operatorname{dim}(\mathfrak{g})+\operatorname{dim}(\mathfrak{g}(l))+\operatorname{dim}\left(\mathfrak{g}\left(l^{\prime}\right)\right)}{2}-\operatorname{dim}(\mathfrak{g}) \\
& =\frac{\operatorname{dim}(\mathfrak{g}(l))+\operatorname{dim}\left(\mathfrak{g}\left(l^{\prime}\right)\right)}{2} \\
& =\frac{\operatorname{dim}(\mathfrak{g})+\operatorname{dim}(\mathfrak{g}(l)) \cap \operatorname{dim}\left(\mathfrak{g}\left(l^{\prime}\right)\right)}{2} \\
& =\frac{\operatorname{dim}(\mathfrak{g})+\operatorname{dim}\left(\mathfrak{g}\left(l+l^{\prime}\right)\right)}{2} .
\end{aligned}
$$

Hence $\mathfrak{b} \cap \mathfrak{b}^{\prime}$ is a polarization at $\ell+\ell^{\prime}$ while $\left\langle\ell+\ell^{\prime},\left[\mathfrak{b} \cap \mathfrak{b}^{\prime}, \mathfrak{b} \cap \mathfrak{b}^{\prime}\right]\right\rangle=0$.

Example 7.2. Let $\mathfrak{g}$ be the Lie algebra spanned by the basis $\left\langle Z_{1}, \cdots, Z_{6}\right\rangle$ and equipped with the brackets

$$
\left[Z_{2}, Z_{3}\right]=Z_{5}, \quad\left[Z_{1}, Z_{4}\right]=Z_{6} .
$$

Let $\ell=Z_{5}^{\star}, \ell^{\prime}=Z_{6}^{\star} \in \mathfrak{g}^{\star}$. Take the polarizations

$$
\mathfrak{b}:=\operatorname{span}\left\langle Z_{6}, Z_{5}, Z_{4}, Z_{3}, Z_{1}\right\rangle
$$

at $\ell$ and

$$
\mathfrak{b}^{\prime}:=\operatorname{span}\left\langle Z_{6}, Z_{5}, Z_{4}, Z_{3}, Z_{2}\right\rangle
$$

at $\ell^{\prime}$.

Then $\mathfrak{b}+\mathfrak{b}^{\prime}=\mathfrak{g}$ and

$$
\mathfrak{b} \cap \mathfrak{b}^{\prime}=\operatorname{span}\left\langle Z_{6}, Z_{5}, Z_{4}, Z_{3}\right\rangle,
$$

which is a polarization at $\ell+\ell^{\prime}$. Hence $\pi_{\ell} \otimes \pi_{\ell^{\prime}} \in \hat{G}$.

\section{REFERENCES}

1. Arnal, D; Fujiwara, H; Ludwig, J. Opérateurs d'entrelacement pour les groupes de Lie exponentiels. (Intertwining operators for exponential Lie groups). (French) Amer. J. Math. 118, No.4, 839-878 (1996) MR.1400061 (98b:22012)

2. Baklouti, A.; Ludwig, J. Désintégration des représentations monomiales des groupes de Lie nilpotents. (Disintegration of monomial representations of nilpotent Lie groups). (French) J. Lie Theory 9, No.1, 157-191 (1999) MR.1680003(2000b:22011)

3. Baklouti, A; Ludwig, J. Entrelacement des restrictions des représentations unitaires des groupes de Lie nilpotents. (Intertwining of restriction of unitary representations of nilpotent Lie groups). (French) Ann. Inst. Fourier 51, No.2, 395-429 (2001) MR1824959 (2002g:22017)

4. Bernat, P; Conze, N; Duflo, M; Levy-Nahas, M; Rais, M; Renouard, P; Vergne, M. Représentations des groupes de Lie résolubles. (French) Monographies de la Société Mathématique de France. 4. Paris: Dunod. X, 272 pp. F 88.00 (1972) MR0444836 (56:3183)

5. Corwin, L; Greenleaf, F.P. Spectrum and multiplicities for restrictions of unitary representations in nilpotent Lie groups. (English) Pac. J. Math. 135, No.2, 233-267 (1988) MR968611 (90b:22011a)

6. Corwin, Lawrence J.; Greenleaf, F.P. Representations of nilpotent Lie groups and their applications. Part 1: Basic theory and examples. (English) Cambridge Studies in Advanced Mathematics, 18. Cambridge University Press. viii, 269 pp. (1990) MR1070979 (92b:22007)

7. Fujiwara, H. Sur les restrictions des représentations unitaires des groupes de Lie résolubles exponentiels. (On the restrictions of unitary representations of exponential solvable Lie groups). (French) Invent. Math. 104, No.3, 647-654 (1991) MR.1106754 (92f:22012) 
8. Fujiwara, H. Représentations monomiales des groupes de Lie nilpotents. (Monomial representations of nilpotent Lie groups). (French) Pac. J. Math. 127, No.2, 329-352 (1987) MR881763 (89c:22015)

9. Kirillov, A.A. Unitary representations of nilpotent Lie groups (Russian, English) Russ. Math. Surv. 17, No.4, 53-104 (1962); translation from Usp. Mat. Nauk 17, No.4(106), 57-110 (1962) MR0142001 (25:5396)

10. Kobayashi, S; Nomizu, K. Foundations of differential geometry. I (English) New York-London: Interscience Publishers, a division of John Wiley \& Sons. XI, 329 pp. (1963) MR0152974 $(27: 2945)$

11. Mackey, G.W. The theory of unitary group representations. (English) Chicago Lectures in Mathematics. Chicago - London: The University of Chicago Press. X, 372 pp. (1976) MR0396826 (53:686)

12. Pukanszky, L. Leçons sur les représentations des groupes (French) Paris: Dunod 1967. VIII, 178 pp. (1967) MR0217220(36:311)

Département de Mathématiques, Faculté des Sciences de Sfax, RTE de Soukra KM 4. B. P. 802,3018 , Sfax, Tunisia

E-mail address: jawhar.abdennadher@fss.rnu.tn

Département de Mathématiques, Laboratoire LMAM UMR 7122, Université de Metz, Ile du Saulcy, F-57045 Metz Cedex 1, France

E-mail address: ludwig@univ-metz.fr 